\title{
Distinguishing electron paramagnetic resonance signature of molecular hydrino
}

\section{Wilfred Hagen ( $\nabla$ W.R.Hagen@tudelft.nl )}

Delft University of Technology; Department of Biotechnology https://orcid.org/0000-0002-1609-6671

\section{Randell Mills}

Brilliant Light Power Inc

\section{Article}

Keywords: hydrino hypothesis, electron paramagnetic resonance, molecular hydrino

Posted Date: February 10th, 2021

DOI: https://doi.org/10.21203/rs.3.rs-144403/v1

License: (c) (i) This work is licensed under a Creative Commons Attribution 4.0 International License. Read Full License 


\title{
Distinguishing Electron Paramagnetic Resonance Signature of Molecular Hydrino
}

\author{
Wilfred R. Hagen ${ }^{1 \bowtie}$, Randell L. Mills ${ }^{2 \bowtie}$
}

${ }^{1}$ Department of Biotechnology, Delft University of Technology, Delft, The Netherlands. ${ }^{2}$ Brilliant Light Power Inc, Cranbury, NJ, USA. ${ }^{凶}$ e-mail: w.r.hagen@tudelft.nl; rmills@brilliantlightpower.com

Summary. Quantum mechanics postulates that the hydrogen atom has a stable ground state from which it can be promoted to excited states by capture of electromagnetic radiation, with the energy of all possible states given by $E_{n}=-13.598 / n^{2} e V$, in which $n \geq 1$ is a positive integer. By contrast, it has been proposed that the $\mathrm{n}=1$ state is not the true ground state, and that so-called 'hydrino' states of lower energy can exist, which are characterized by fractional quantum numbers $\mathrm{n}=1 / \mathrm{p}$, in which $1<\mathrm{p} \leq 137$ is a limited integer ${ }^{1,2}$. Electron transition to a hydrino state, $\mathrm{H}(1 / \mathrm{p})$ is non-radiative and requires a quantized amount of energy, $2 \mathrm{mE}_{1}$ ( $\mathrm{m}$ is an integer), to be transferred to a catalyst ${ }^{3,4}$. Since its inception ${ }^{5}$ the hydrino hypothesis has remained highly controversial $^{6-17}$ and laboratory verification studies by its proponents have been criticised ${ }^{18,19}$. Remarkably, no experimental testing by independent researchers has been described in the literature over the past 31 years. Here, we give an account of an independent electron paramagnetic resonance (EPR) study of molecular hydrino $\mathrm{H}_{2}(1 / 4)$ that was produced by a plasma reaction of atomic hydrogen with non-hydrogen bonded water as the catalyst. A sharp, complex, multi-line EPR spectrum is found, whose detailed properties prove to be semiquantitatively consistent with predictions ${ }^{20}$ from hydrino theory with an average error less than $0.09 \mathrm{G}(0.2 \%)$ over a $39 \mathrm{G}$ span of 37 lines. We have sought but failed to find reasonable alternative, 'conventional' interpretations for the detected paramagnetism. Fundamental relevance of the hydrino hypothesis lies in its challenging some of the foundations of the theory of quantum mechanics ${ }^{1}$. Very high net energy release during hydrino formation signifies technological relevance as a novel method of green energy production with recent validation at the $100 \mathrm{~kW}$ continuous power level by measurement of steam production ${ }^{20-27}$. 
Introduction. The quantized energy levels of the hydrogen atom are $E_{\mathrm{n}}=-13.598 / \mathrm{n}^{2} \mathrm{eV}$, in which the principal quantum number $\mathrm{n}$ is a positive integer. The electronic ground state has $\mathrm{n}=1$. Higher states can be populated by absorption of light according to the Rydberg equation $v=$ $\mathrm{R}_{\mathrm{H}}\left[\left(1 / \mathrm{n}_{2}\right)-\left(1 / \mathrm{n}_{1}\right)\right]$ with $\mathrm{R}_{\mathrm{H}}=109.677 \mathrm{~cm}^{-1}$. R. Mills has hypothesized and experimentally tested that the $\mathrm{n}=1$ state is not the absolute ground state and that lower-energy 'hydrino' states characterized by fractional quantum numbers $1 / \mathrm{p}$, with $2 \leq \mathrm{p} \leq 137$, can exist ${ }^{1,2,20,28}$, and, furthermore, that $\mathrm{H}(1 / \mathrm{p})$ can be produced from $\mathrm{H}(\mathrm{n}=1)$ in a non-radiative process whereby a catalyst reversibly takes up an amount of energy equal to $(\mathrm{p}-1) \times 27.196 \mathrm{eV}$ such that an total amount equal to $\left(\mathrm{p}^{2}-1\right) \times 13.598 \mathrm{eV}$ is ultimately released as heat ${ }^{4,20,28}$. These proposals have been presented as elements of a contentious theory of much wider coverage, called the grand unified theory of classical physics (here abbreviated as GUTCP) with the much wider claim to revisit the foundations of quantum mechanics ${ }^{1,20,29}$. Although a critical appraisal of this controversial assertion on theoretical grounds initially developed in journals of established reputation ${ }^{6,7,9,10}$, subsequently the works of independent opponents ${ }^{8,11}$, neutral observers ${ }^{14}$, and adherents ${ }^{13,15,17}$ alike have slipped off either to publications with impact factors typically well below unity or to un-reviewed papers. Thus, the present verdict of the scientific community at large appears to be one of disregard, if not disdain.

Worthy of note is the fact that all cited evaluations by independent researchers thus far are exclusively concerned with theoretical arguments, while occasional independent experimental testing of the theory's predictions has not been published and has only been indirectly cited ${ }^{14}$. Moreover, all of these criticisms are based on the incompatibility of hydrinos with quantum mechanics with the inherent assumption of the validity of quantum mechanics, which is circular reasoning. Mills' prediction of hydrino states of hydrogen is not based on quantum mechanics. It is based on physical laws, and the resulting physical theory is remarkably predictive over 85 orders of magnitude of scale in exact solutions having fundamental constants only ${ }^{1,20}$. Since Mills theory is physical/testable, we consider the bias away from experimental testing and reporting as undesirable in view of the potentially far reaching fundamental and technological implications of the GUTCP. The present study is an attempt to break a 30-year independent testing silence by providing an objective and readily reproducible spectroscopic test on a system allegedly containing molecular hydrino, $\mathrm{H}_{2}(1 / 4)$. This work is not a test of the GUTCP as a whole; it has a 
bearing on three sub-aspects: hydrino existence, catalytic hydrino formation, and paramagnetic properties of $\mathrm{H}_{2}(1 / 4)$ predicted by the theory.

Sample production. A common feature of a hydrino state with an excited $\mathrm{H}$ state is that both comprise an electron, a proton, and a photon. In an excited state, the photon superimposes the proton field to decrease the central field at the electron to $+\mathrm{e} / \mathrm{n}$ ( $\mathrm{e}$ is the fundamental charge) and creates a radial dipole instability that results in radiation. Conversely, the photon of a hydrino state increases the central field at the electron to $+(1+\mathrm{m})$ e and creates a radial monopole that is radiatively stable. According to Mills ground-state $(n=1)$ atomic hydrogen can be converted to atomic hydrino $(n=1 /(1+m))$ by means of a nonradiative resonant energy transfer to a catalyst with potential energy $=\mathrm{m} \times 27.2 \mathrm{eV}$ (that is $\left.2 \mathrm{~m} \times \mathrm{E}_{1}\right)$ according to the reaction

$$
\mathrm{m} \times 27.2 \mathrm{eV}+\mathrm{H}(1)+\mathrm{Cat} \rightarrow \mathrm{H}^{*}(1 /(1+\mathrm{m}))+\mathrm{Cat}^{*}+\mathrm{m} \times 27.2 \mathrm{eV}
$$

in which the energy term on the left is energy absorbed by the catalyst (typically by resonant ionization) and the term on the right is the energy released by the increase in the potential energy of the hydrogen atom to form $\mathrm{H}^{*}(1 /(1+\mathrm{m}))$, an intermediate of the hydrino atom of radius $\mathrm{a}_{\mathrm{H}}$. Subsequently the ionized catalyst, Cat*, regenerates by recombination, with the release of its previously gained ionization energy, and the hydrino intermediate converts to stable $\mathrm{H}(1 /(\mathrm{m}+1))$ having a radius of $\mathrm{a}_{\mathrm{H}} /(1+\mathrm{m})$ by release of additional energy such that the overall release of energy is $\left[(\mathrm{m}+1)^{2}-1\right] \times 13.6 \mathrm{eV}$. By considering quantum state $\mathrm{p}=\mathrm{m}+1$ the reaction may be written

$$
\mathrm{H}(1) \rightarrow \mathrm{H}(1 / \mathrm{p})+\left(\mathrm{p}^{2}-1\right) \times 13.6 \mathrm{eV}
$$

The Rydberg formula with the inclusion of the hydrino states is

$$
\mathrm{E}=13.6 \mathrm{eV} / \mathrm{n}^{2} ; \mathrm{n}=1 / 137,1 / 136 \ldots 1 / 4,1 / 3,1 / 2,1,2,3 \ldots
$$

The hydrino transition reaction requires atomic $\mathrm{H}$ and a single catalyst species which is typically formed chemically or by a plasma reaction ${ }^{20,28}$. Further reactivity produces molecular hydrino 
$\mathrm{H}_{2}(1 / \mathrm{p})$ from atomic hydrino $\mathrm{H}(1 / \mathrm{p})$ when the bond energy is removed by collision with a third body, which can be a reactor-wall constituent $\left(\mathrm{cf}^{30}\right)$. A variety of species can resonantly and nonradiatively accept $\mathrm{m} \times 27.2 \mathrm{eV}$ from atomic hydrogen to serve as catalyst for hydrino formation (Ref-1, Chp $5 \&^{20}$ ); in the present case we use the nascent (that is, in situ prepared, not hydrogen-bonded) water molecule with potential energy $3 \times 27.2 \mathrm{eV}$ wherein the solutions of the water molecule and hydrogen bonded water molecules were given previously (Ref-1, Chps13 and 16). Details of the sample preparation are given in the METHODS section. Briefly, the reactor is a closed vessel in which a low-voltage discharge is created between a liquid gallium electrode and a solid tungsten electrode with water and hydrogen introduced from a supported- $\mathrm{Pt}_{2} / \mathrm{O}_{2}$ recombiner supplied with $\mathrm{H}_{2}$ gas and trace $\mathrm{O}_{2}$ to form trace nascent or non-H-bonded water catalyst. Either additional oxygen or water vapor are introduced to produce gallium oxide that is collected. After dissolution of the gallium oxide in $4 \mathrm{M} \mathrm{KOH}$, a unique non-soluble product comprising $\mathrm{Ga}(\mathrm{O}) \mathrm{OH}$ in the form of an aggregate of micro-spheres containing molecular hydrino $\mathrm{H}_{2}(1 / 4)$ slowly polymerizes as shown by scanning electron microscopy (SEM) and transmission electron microscopy (TEM) in Fig. 1. Energy dispersive X-ray spectroscopy (EDS) showed an elemental composition of $\mathrm{GaO}_{2.1}$ (Extended Data Fig. 1). Rutherford backscattering spectrometry (RBS) performed on the GaOOH: $\mathrm{H}_{2}(1 / 4)$ identified the composition as $\mathrm{GaO}_{1.68} \mathrm{H}_{1.32}$ with a density of $8.56 \times 10^{22}$ atoms $/ \mathrm{cm}^{2}$ corresponding to an excess $\mathrm{H}$ content, some of which is hydrino hydrogen based on the results of EPR reported herein and other analytical tests reported by Mills et al. ${ }^{20,28}$. Time-of-flight secondary ion mass spectrometry (ToF-SIMS), presented in Extended Data Fig. 2, showed $\mathrm{Ga}$ in the positive ion spectrum and $\mathrm{O}$ and $\mathrm{H}$ as dominant ions in the negative ion spectrum wherein the hydride ion was elevated compared to control $\mathrm{GaOOH}$. No hydrocarbons above adventitious levels were present and no nitrogen was found indicating the unlikeliness for EPR signals to originate from organic radicals. Equally, in the positive spectrum no potentially paramagnetic transition ions were present. Selected area electron diffraction (SAED) with the transmission electron microscope (Extended Data Fig. 3) revealed the samples to comprise two different morphological and crystalline forms of $\mathrm{GaOOH}$ : rods with orthorhombic diffraction pattern matched control $\mathrm{GaOOH}$, which lacks molecular hydrino, in morphology and crystalline structure ${ }^{31}$, and were not sensitive to the TEM electron beam; on the other hand, morphologically polymeric crystals comprising hexagonal crystalline structure were very electron-beam sensitive, and were assigned to novel $\mathrm{GaOOH}: \mathrm{H}_{2}(1 / 4)$. X-ray diffraction 
(XRD) showed a phase shift from the $\mathrm{GaOOH}$ control lines with different deviations between $\mathrm{NaOH}$ and $\mathrm{KOH}$ formed $\mathrm{GaOOH}: \mathrm{H}_{2}(1 / 4)$ as illustrated in Extended Data Fig. 4.

Paramagnetism. Extensive background information for this paragraph is provided in Supplementary information ${ }^{20}$. Alternative to the probabilistic matter waves of quantum mechanics, the electron in a hydrogen atom is modelled in GUTCP as a two-dimensional spherical membrane of infinitesimal thickness in which current flows along two infinite, nested rotation sets of great circle filaments. This current pattern naturally gives rise to both orbital and spin angular momentum wherein the latter defines a $g$ factor equal to ${ }^{29} 2+0.0023193$. In the hydrogen molecule the spherical current pattern becomes a prolate spheroid in which the pairing of two electrons leads to a diamagnetic ground state. Atomic hydrino differs from $\mathrm{H}(\mathrm{n}>1)$ states in that rather than the absorption of a photon to form an excited state, $\mathrm{H}(\mathrm{n}=1 / \mathrm{p})$ it is formed by a non-radiative energy transfer to a resonant energy acceptor followed by continuum extreme ultraviolet radiation to the final stable hydrino atomic state. The continuum EUV radiation was recorded in the laboratory at the $20 \mathrm{MW}$ optical power level with a predicted 10.1 shortwave cutoff, and this radiation is observed astrophysically over all space ${ }^{20,32,33}$. Two hydrino atoms react to form molecular hydrino having two photons that are phase-locked to the electron current and circulate in opposite directions. Consequently, the molecule has a diamagnetic and a paramagnetic electron, the latter with g equal to $2+2 \times 0.0023193=2.0046386$ (Ref- 1 , Chp16 $\left.\&^{20,28}\right)$. This fundamental prediction from first principles provides a simple and accurate testing criterion for the existence of molecular hydrino.

EPR spectroscopy. A wide magnetic field scan EPR spectrum of the $\mathrm{Ga}(\mathrm{O}) \mathrm{OH}$ solid powder taken at ambient temperature, exhibits a single derivative feature only against an essentially flat background, and with g value close to the free-electron value (Fig. 2a). Zooming-in on this feature (Fig. 2b) shows it to consist of two separate lines plus multiple weak signals in the lowand high-field wings. The center of the two main lines corresponds to an apparent $g$ value of 2.0045(6) which is close to the value of 2.00464 predicted for the $\mathrm{H}_{2}(1 / 4) \mathrm{S}=1 / 2$ spin-only doublet system. The two lines are separated by circa 4 Gauss and are of equal intensity. 
Microwave power saturation plots (Extended Data Fig. 5) are very similar for the two peaks and are consistent with inhomogeneous broadening ${ }^{34}$ (see below).

Concentrating on resolving fine structure in the main peaks we reduce the magnetic-field modulation amplitude to $25 \mathrm{mGauss}$ (that is, below the bandwidth of the $100 \mathrm{kHz}$ modulation frequency). The spectral amplitude in a single scan drops to below a signal-to-noise ratio of unity and extensive averaging over six hours and filtering is required to afford the high-resolution pattern in Fig. 2c. Each line has resolved in an isotropic equidistant beat pattern with sub-line separation of circa 0.32 Gauss.

For individual sub-lines we observe an apparent peak-to-peak width of circa 170 mGauss which is highly unusual for solid-state samples. Such narrow lines have been found for (i) organic radicals in organic solvents at ambient temperature ${ }^{35}$; (ii) small paramagnetic molecules in matrices of noble gasses solidified at cryogenic temperatures ${ }^{36}$; (iii) single hydrogen atoms encapsulated in molecular cages ${ }^{37}$; and (iv) paramagnetic molecules in the gas phase at low pressure $^{38}$. Excluding the first two options on obvious grounds (no organic solvents and no cryogenic temperatures) and the third one on spectroscopic grounds (atomic hydrogen EPR is a single line at the free electron $g$ value split widely by proton hyperfine interaction), the narrow line width that we observe would be consistent with the detection of a low-pressure paramagnetic gas occluded in a solid.

We recorded the spectrum in Fig. 2d under optimized conditions for the detection and resolution of satellite lines whose existence was indicated by the small periodic peaks in the wings of the spectrum in Fig. 1b. Thus, the fine structure of the two central lines was slightly deformed by over-modulation and by mild microwave power saturation, and the data collection was extended to 40 hours with constant frequency monitoring for subsequent correction of individual 4-min traces for minor frequency drift.

In spin quantification (METHODS) we find that a complete spectrum of high resolution, such as in Fig. 2d, represents an $\mathrm{S}=1 / 2$ concentration of circa $2.6 \mu \mathrm{M}$ if the paramagnet would be homogeneously distributed over the sample volume. Transmission electron microscopy (Fig 1) and XRD show the $\mathrm{Ga}(\mathrm{O}) \mathrm{OH}$ polymer to comprise micro-spherical particles of the order of 100 $\mathrm{nm}$ diameter with an estimated spatial occupancy of roughly $10 \%$. This would make the actual concentration of the $\mathrm{H}_{2}(1 / 4)$ gas in occlusion very approximately $26 \mu \mathrm{M}$, which is equivalent to a partial pressure of circa $6 \times 10^{-4}$ bar, qualitatively consistent with the observed narrow EPR line 
width $^{38,39}$. Even if regular $\mathrm{H}_{2}$ would co-occlude at, say, atmospheric pressure the small cross section for collision of molecular hydrino $\mathrm{H}_{2}(1 / 4)$ would ensure a low collision frequency in agreement with the observed line width.

Simulation of the fine structure in spectrum Fig. $2 \mathrm{c}$ indicated the line shape to be Gaussian within the limitation set by the overlap of individual lines. The sets of satellite lines are better separated, and analysis of the first down-field triplet clearly shows the line shape to be essentially Gaussian (Extended Data Fig 2). This implies inhomogeneous broadening, consistent with the power-saturation analysis above, and could be caused by interaction of hydrino molecules with the inner 'wall' of the inorganic polymer cage. In turn, this would imply the real lifetime line width from gas collision to be significantly less than the observed 170 mGauss inhomogeneous line width.

EPR interpretation. Supplementary information provides detailed theoretical background on EPR line assignments ${ }^{20}$. Molecular hydrino comprises two protons at the foci of a two-electron prolate spheroid molecular orbital membrane, and an absorbed photon. The latter splits into two photons that are phase locked with the oppositely directed current patterns of the two electrons each consisting of an angularly distributed infinite ensemble of closed grand ellipse filaments of moving charge ${ }^{1}$ of an equipotential, minimum energy membrane surface. Under this model exact solutions of a fine structure in the EPR ensues with parameters whose predicted magnitudes (Ref1, Chp 16 and $^{20,28}$ ) can be tested against experimental values.

The unique electronic structure results in one paramagnetic and one diamagnetic electron. The former induces a current in the latter by means of spin-orbit coupling resulting in a split of the original resonance into two lines separated by a frequency-independent interaction, which is for $\mathrm{H}_{2}(1 / 4)$ predicted to be of magnitude 3.9943 Gauss with the field center of the two lines corresponding to the original $\mathrm{g}$ value of ${ }^{20} 2.00464$. Experimentally we observe two lines of equal intensity separated by 3.9 Gauss whose center is found at $\mathrm{g}=2.0045(6)$.

Linkage of magnetic flux by the electron membrane is quantised in units of the magnetic flux quantum $\varphi_{0}=\mathrm{h} / 2 \mathrm{e}$, which results in a sub-line pattern of each of the two main lines with a predicted separation of 0.311 Gauss $^{20}$. The observed separation is 0.32 Gauss. Similar to the case of excited-states of the regular $\mathrm{H}_{2}$ molecule, the two electrons in $\mathrm{H}_{2}(1 / 4)$ may rotate relative to 
each other along the semimajor axis during a spin transition. The relative rotation is quantized in terms of $\mathrm{m}$ integer units of $\hbar$ in opposite directions with the spin-orbit splitting in frequencyindependent field units equal to \pm m times twice the splitting between the two main lines, that is $\pm \mathrm{m} \times 7.9885$ Gauss. Additionally, the unpaired electron must link the magnetic flux component corresponding to spin-orbit coupling. This flux contribution increases the magnetic energy and the energy of the combined spin flip and spin-orbit coupling transition energy for a given spinorbital quantum number $\mathrm{m}$. Thus the downfield spin-orbital splitting peaks are shifted further downfield by the corresponding magnetic energies, whereas the upfield spin-orbital splitting peaks are not shifted since they correspond to emission of the spin-orbital coupling transition energies alone.

Furthermore, each of these satellite lines is split through the linkage of magnetic flux during a spin transition, and the exact solution of the splitting is circa 0.62 Gauss for $|\mathrm{m}|=1$ and circa 0.93 for $|\mathrm{m}|>1$ where the latter lines follow an intensity pattern ${ }^{20} \mathrm{I}_{\mathrm{m}+1} / \mathrm{I}_{\mathrm{m}}=\mathrm{m} /(\mathrm{m}+2)$. The predicted details ${ }^{20}$ of this complex pattern of split satellite lines asymmetrically grouped around a $\mathrm{g}$ value of 2.00464 make up a stick spectrum that, when convoluted with a Gaussian derivative, forms a semi-quantitative reproduction (Fig. 2e) of the experimental spectroscopy (see also Supplementary information Table-4).

Consistency controls. Unequivocal interpretation of complex EPR spectra typically requires analysis of data taken at more than one microwave frequency. The magnetic model of molecular hydrino $\mathrm{H}_{2}(1 / 4)$, providing a basis for interpretation of the EPR, predicts a number of features to be either dependent or independent of microwave frequency. These predictions can be checked in separate experiments as consistency tests. The g value of 2.00464 in between the two main lines is a real $\mathrm{g}$ value and thus its field position should be linear in the microwave frequency. Contrarily, all fine structure splittings are predicted to be constant in field units and thus independent of the frequency.

As a check we have taken data in Q-band at circa $35 \mathrm{GHz}$. Here, practical complications arise resulting in reduced signal-to-noise ratios. For $S=1 / 2$ systems, any spectrometer operating in a frequency band different from X-band is generally found to exhibit a significantly lower concentration sensitivity. Furthermore, the maximal applicable intensity of the microwave is 
found to be limited (that is, the spectrometer is not tunable at higher microwave powers) apparently due to a relatively high dielectric permittivity of the $\mathrm{Ga}(\mathrm{O}) \mathrm{OH}$ samples. Fig 3a shows two traces resulting from extensive averaging, one taken under over-modulating conditions to emphasize the main two-line pattern, and one taken at lower modulation amplitude in an attempt to resolve fine structure. Consistent with the interpretation of the $\mathrm{X}$-band spectrum we find a doublet of lines whose spectral center has a real $g$ value of 2.0046 and with a frequencyindependent splitting of circa 4 Gauss. Under the employed conditions, the underlying broad signal has turned dispersive and thus shows up as an absorption-shape feature. A lower modulation amplitude does not afford resolution of the two-lines' fluxonal fine structure, which indicates that the spectral line width has increased with frequency. This is in fact consistent with our previous conclusion (cf. Fig 2c and Extended Data Fig. 6) that the line shape is Gaussian due to inhomogeneous broadening, which implies a line width in field units linear in the frequency ${ }^{40}$.

Since the signal-to-noise ratio in Q-band was insufficient to detect the satellite lines, and since attempts to measure the samples in other frequency bands were hitherto unsuccessful (not shown), we took data at two, well-separated frequencies within the X-band thus allowing for comparison of high-resolution spectra with the trade-off of reduced frequency resolution (Fig. 3bc). Data taken at $9.46 \mathrm{GHz}$ were transformed for comparison with data taken at $9.85 \mathrm{GHz}$ in two ways: (1) frequency-ratio conversion of every digital point of the field axis, and (2) singlevalued overall field shift to create maximal overlap of the two spectra. In the first method all real g values will overlay while features constant in the field will mismatch. In the second method all features of a fine-structure pattern constant in the field will overlay when the selected field point of conversion corresponds to the $\mathrm{g}$ value of that pattern. Fig. $3 \mathrm{~b}$ gives the result of the first method: all features mismatch except for the spectral center at $\mathrm{g}=2.0046$, therefore the latter is the only real g value and all other features are from frequency-independent hyperfine interactions. Fig $3 \mathrm{c}$ gives the result of the second method: all lines match, including all satellite lines and all fluxon sub-lines of the two main lines, therefore all features are from frequencyindependent hyperfine interactions and they all share a single, common g value. The spectral features assigned to $\mathrm{H}_{2}(1 / 4)$ were repeated in duplicate at the spectrometer manufacturer location (Bruker Scientific LLC, Bileria, MA) using two stations, EMXnano and EMXplus instruments ${ }^{20}$.

Moreover, Raman, and electron-beam excitation spectra show the same spin-orbital coupling and fluxon linkage splittings as EPR in energy ranges that differ by reciprocal of the 
$\mathrm{H}_{2}(1 / 4)$ diamagnetic susceptibility coefficient: $1 / 7 \times 10^{-7}=1.4 \times 10^{6}$, wherein the induced diamagnetic orbital magnetic moment active during EPR was replaced by the orbital molecular rotational magnetic moment active during Raman and electron-beam excitation of rotational transitions ${ }^{20}$. It is also remarkable, that ten Raman lines recorded on $\mathrm{GaOOH}: \mathrm{H}_{2}(1 / 4): \mathrm{H}_{2} \mathrm{O}$ match those of Diffuse Interstellar Bands (DIBS) ${ }^{20}$. Hydrino is consistent with observations that implicate that the identity of dark matter is an unusual state of hydrogen ${ }^{20,33}$.

Alternative interpretation. Alternative to the hydrino analysis in Fig $2 \mathrm{e}$ the spectrum in Fig $2 \mathrm{~d}$ can also be approximately reproduced under a conventional phenomenological spin Hamiltonian assuming an unusual combination of two isotropic radicals of unequal intensity each with a $\mathrm{g}$ value of 2.0046. This model would require the two main lines to be due to an isotropic $S=1 / 2$ system split by an $\mathrm{I}=1 / 2$ nucleus with $\mathrm{A}_{\text {iso }} \approx 3.9$ Gauss with additional hyperfine structure form a combination of some 5 nuclei the majority of which has also $I=1 / 2$. A second $S=1 / 2$ system should give rise to the satellite lines due to a different combination of some 5 nuclei, one of which should have I = $1\left(\right.$ e.g. ${ }^{14} \mathrm{~N}$ ) to account for the repeating triplet pattern (see Extended Data Fig. 7 for a detailed analysis).

We consider this alternative explanation of the EPR highly unlikely on the following grounds. The reaction mixture only contains $\mathrm{H}_{2}, \mathrm{O}_{2}, \mathrm{H}_{2} \mathrm{O}$, and $\mathrm{Ga}$. Even in the presence of trace contaminants of, e.g., $\mathrm{C}, \mathrm{N}$, we cannot envision how the high-temperature plasma reaction conditions and sample formation in strong aqueous base could lead to the formation of stable radical structures of considerable complexity. The ToF-SIMs, EDS, and XRD analyses also eliminate alternatives. Furthermore, since the sample is a solid, for complex radicals one would expect to see anisotropy in the spectra. In particular absorption-shaped peaks that come with axial or rhombic symmetry of the spin Hamiltonian are not observed.

Unsolved problems. The GUTCP fit to the X-band spectrum of $\mathrm{H}_{2}(1 / 4)$ is semi-quantitative with an average error of $0.096 \mathrm{G}$ over the 11 lines assigned to spin-orbital coupling splitting. The actual positions of the satellite lines slightly deviate from their predicted values (Fig. 2de and Supplementary information Table-4). Also, the fluxon separation for any given position does not quantitatively fit the predicted value; in particular the separation is not a constant. Also, the 
number and relative intensities of fluxon lines for a given satellite line are presently not understood. Possibly these 'irregularities' are caused by interactions of the gaseous $\mathrm{H}_{2}(1 / 4)$ with the wall of the polymeric $\mathrm{Ga}(\mathrm{O}) \mathrm{OH}$ microspheres.

A broad signal underlies the molecular-hydrino assigned spectrum. Its spectral center corresponds to the $\mathrm{g}$ value of 2.0046 within experimental error. Its temperature behaviour is very different from that of the hydrino-assigned spectrum (Extended Data Fig. 8). The origin and nature of the broad signal are presently unknown, however, a reasonable hypothesis would be to assume that there are two phases of $\mathrm{GaOOH}$ that encapsulate $\mathrm{H}_{2}(1 / 4)$ wherein $\mathrm{H}_{2}(1 / 4)$ is a near free gas in only one phase $\mathrm{e}^{20,28}$. A scanning/transmission electron microscope (SEM/TEM) used for imaging and selected area electron diffraction (SAED) (Extended Data Fig. 3) showed that the $\mathrm{GaOOH}: \mathrm{H}_{2}(1 / 4)$ sample comprised two different morphologically polymeric crystals of $\mathrm{GaOOH}$, a hexagonal crystalline structure that was very sensitive to the TEM electron beam, and rods having orthorhombic crystalline structure that were not electron beam sensitive. The rod crystal morphology and crystalline structure match those of the literature for control $\mathrm{GaOOH}$ that lacks gaseous molecular hydrino inclusion ${ }^{31}$. The XRD crystal system for Tsumgallite (control GaOOH) is orthorhombic. The hexagonal phase is likely the source of the fine structure EPR spectrum, and the orthorhombic phase is likely the source of the broad background EPR feature. Cooling may selectively eliminate, e.g., by microwave power saturation, the observed near free-gas-like EPR spectral behavior of $\mathrm{H}_{2}(1 / 4)$ trapped in the hexagonal crystalline matrix. In addition to wall interactions, deviations from theory could be due to the influence of the proton of $\mathrm{GaOOH}$ and those of absorbed water. Also, matrix orientation in the magnetic field, matrix interactions, and interactions between one or more $\mathrm{H}_{2}(1 / 4)$ could cause some shifts.

Deuterium substitution was performed to eliminate an alternative assignment of any EPR spectral lines as being nuclear split lines. The deuterated analog of $\mathrm{GaOOH}: \mathrm{H}_{2}(1 / 4)$, GaOOH:HD(1/4), was confirmed by Raman spectroscopy ${ }^{20,28}$. The EPR spectrum of the deuterated analog showed a singlet with no fine structure; thus, eliminating any possible nuclear splitting assignment. The $\mathrm{g}$ factor and profile matched that of the singlet of $\mathrm{GaOOH}: \mathrm{H}_{2}(1 / 4)$ wherein the singlet in both cases was assigned to the orthorhombic phase. The XRD of the deuterated analog matched that of the hydrogen analog, both comprising gallium oxyhydroxide. TEM confirmed that the deuterated analog comprised $100 \%$ orthorhombic phase $\left(\mathrm{cf}^{31}\right)$. The phase preference of the 
deuterated analog may be due to a different hydrino concentration and kinetic isotope effect which could have also reduced the concentration.

Lastly, further investigation is warranted to assign a peak slightly downfield from the central $g$ value of 2.0046 the X-band spectra having a small signal of apparent axial symmetry (cf Fig. 2d). This peak is likely the pure spin-flip (no spin orbital coupling or fluxon linkage splitting) transition peak of the orthorhombic $\mathrm{GaOOH}$ phase with entrapped $\mathrm{H}_{2}(1 / 4)$ molecules that are constrained relative to the free gas state or near free gas state of the hexagonal phase.

Conclusions. A plasma reaction has been carried out intended to produce molecular hydrino using non hydrogen bonded water as the catalyst and with liquid gallium as one of the electrodes. Polymeric $\mathrm{Ga}(\mathrm{O}) \mathrm{OH}$ with a spherical particle structure, presumably containing $\mathrm{H}_{2}(1 / 4)$, was purified from the reaction mixture. $\mathrm{H}_{2}(1 / 4)$ is proposed to be an $\mathrm{S}=1 / 2$ paramagnet with complex fluxonal and spin-orbital coupling level structure. The solid $\mathrm{Ga}(\mathrm{O}) \mathrm{OH}$ compound exhibits a complex gas-phase-like X-band EPR spectrum at ambient temperature whose fine structure semi-quantitatively agrees with hydrino-theory predictions. This analysis is consistent with frequency-dependent studies, while alternative, conventional interpretations are judged to be extremely unlikely. In summary, the present study provides compelling EPR spectroscopic evidence for the existence of hydrino. In view of the possible far-reaching implications of this conclusion for the theory of quantum mechanics, for hydrogen-related chemistry, for astronomy, and for energy transduction and production technology (Refs 1,20 and references therein), it is also offered as an urgent invitation to academia at large to repeat and extend the described experiments in lieu of refutation on quantum mechanical theoretical grounds. 


\section{REFERENCES}

1. Mills, R.L. The grand unified theory of classical physics, three volumes. brilliantlightpower.com/book-download-and-streaming / (2020).

2. Mill, R.L. The hydrogen atom revisited. Int. J. Hydrogen Energy 25, 1171-1183 (2000).

3. Mills, R.L., et al. Commercializable power source using heterogeneous hydrino catalysts. Int. J. Hydrogen Energy 35, 395-419 (2010).

4. Mills, R., Lotoski, J., Good, W. \& He, J. Solid fuels that form $\mathrm{HOH}$ catalyst. Int. J. Hydrogen Energy 39, 11930-11944 (2014).

5. R. L. Mills and S. Kneizys, "Excess heat production by the electrolysis of an aqueous potassium carbonate electrolyte and the implications for cold fusion”, Fusion Technol. 20, 65-81, (1991).

6. Reichhardt, T. Out of this world. Nature 420, 10-11 (2002).

7. Rathke, A. A critical analysis of the hydrino model. New J. Phys. 7, 127 pp 1-9 (2005).

8. $\quad$ Connett, J.E. \& Zimmerman, P.D. Against hydrinos. Phys. World 18, 21 (2005).

9. $\quad$ Dombey, N. The hydrino and other unlikely states. Phys Lett. A 360, $62-65$ (2006).

10. de Castro, A.S. Orthogonality criterion for banishing hydrino states from standard quantum mechanics. Phys Lett. A 369, 380-383 (2007).

11. Guizzo, E. Hot or not? IEEE Spectrum 46, 36-38 (2009).

12. Loureiro, J, \& Amorim, J. Possibility of nonexistence of hot and superhot hydrogen atoms in electrical discharges. Phys. Rev. E 82, 035401 (2010).

13. Naudts, J. On the hydrino state of the relativistic hydrogen atom. arXiv:physics/0507193v2 (2005).

14. Rodgers, P. Hydrogen results cause controversy. Phys. World 18, 12-13 (2005).

15. Bourgoin, R.C. Inverse quantum mechanics of the hydrogen atom: a general solution. Adv. Studies. Theor. Phys. 1, 381-393 (2007).

16. Phillips, J. Selective atomic hydrogen heating in plasmas: implications for quantum theory. Int. J. Hydrogen Energy 34, 9816-9823 (2009).

17. Selke, D.L. Against point charges. Appl. Phys. Res. 7, 138-139 (2015).

18. Phelps, A.V. \& Clementson, J. Interpretation of EUV emissions observed by Mills et al. Eur J. Phys. D 66, 120 pp 1-4 (2012). 
19. Lawler, J.E. \& Goebel, C.J. Comment on "Time-resolved hydrino continuum transitions with cutoffs at $22.8 \mathrm{~nm}$ and $10.1 \mathrm{~nm}$ ". Eur. J. Phys. D 66, 29 pp 1-2 (2012).

20. Mills, R. L., Dong, Z., Jenkins, J. Gandhi, R., Mehta, N.S., Mhatre, S, Sharma, P., "Hydrino states of hydrogen", Supplementary information to the present paper, https://brilliantlightpower.com/hydrino-states-of-hydrogen/.

21. Nansteel, M.W., "Water bath calorimetry (120420): report”, https://brilliantlightpower.com/pdf/Report on Water Bath Calorimetry 12.04.20.pdf.

22. Nansteel, M.W., "Plasma cell water bath calorimetry: data and analysis (March 11, 2020)", https://brilliantlightpower.com/pdf/Waterbath Calorimetry Data and Analysis 031120. pdf.

23. Nansteel, M.W., "Molten metal plasma cell calorimetry: data and analysis (January, 2020)",

https://brilliantlightpower.com/pdf/Nansteel_Molten_Metal_Calorimetry_Data_and_Anal ysis_Jan_2020.pdf.

24. Booker, R., "Report on the power output of Liquid gallium SunCells at Brilliant Light Power”, https://brilliantlightpower.com/pdf/Randy_Booker_Report.pdf.

25. Tse, S.D., "Consultant report on onsite molten gallium metal and water bath calorimetry”, https://brilliantlightpower.com/pdf/Tse-Validation-Report-Brilliant-Light-Power.pdf.

26. Nansteel, M.W, "Report on Parr calorimetry experiments conducted February-March, 2019, https://brilliantlightpower.com/wpcontent/uploads/pdf/Calorimetry_Validation_Report-3.2019.pdf.

27. Nansteel, M.W. "Report on BLP Spectroscopy Experiments Conducted on October 6, 2017”, https://brilliantlightpower.com/pdf/Spectroscopy_Nansteel_Report_040219.pdf.

28. Mills, R.L., "Techniques and unique and characteristic signatures to identify Hydrino are predicted from exact closed-form solutions of atoms and molecules", https://brilliantlightpower.com/pdf/Analytical Presentation.pdf.

29. Mills, R.L. The grand unified theory of classical quantum mechanics. Int J. Hydrogen Theory 27, 565-590 (2002).

30. Poole, H.H. Atomic hydrogen III-The energy efficiency of atom production in a glow discharge. Proc. Roy Soc. 163, 424-454 (1937). 
31. Li, S.-J., Zheng, C. \& Lobring, K.C. Z. Kristallogr. NCS 218, 11-12 (2003).

32. R. Mills, Y. Lu, R. Frazer, "Power Determination and Hydrino Product Characterization of Ultra-low Field Ignition of Hydrated Silver Shots”, Chinese Journal of Physics, Vol. 56, (2018), pp. 1667-1717.

33. R. Mills, J. Lotoski, Y. Lu, "Mechanism of soft X-ray continuum radiation from lowenergy pinch discharges of hydrogen and ultra-low field ignition of solid fuels", Plasma Science and Technology, Vol. 19, (2017), pp. 1-28.

34. Portis, A.M. Electronic structure of F centers: saturation of the electron spin resonance. Phys. Rev. 91, 1071-1078 (1953).

35. Jones, M.T. Electron spin resonance absorption of tris-p-nitrophenylmethyl. J. Chem. Phys. 35, 1146 (1961).

36. Feldman, V.I., Sukhov, F.F. \& Orlov, A.Y. Hydrogen atoms in solid xenon: trapping site structure, distribution, and stability as revealed by EPR studies in monoisotopic and isotopically enriched xenon matrices. J. Chem. Phys, 128, 214511 (2008).

37. Päch, M. \& Stösser, R. Scavenger assisted trapping of atomic hydrogen in $\mathrm{Si}_{8} \mathrm{O}_{12}$ cages. $J$. Phys. Chem. A 101, 8360-8365 (1997).

38. Beringer, R. \& Castle, J.G. Microwave magnetic resonance spectrum of oxygen. Phys. Rev. 81, 82-88 (1951).

39. McDonald, C.C. Multiple-quantum transitions in EPR spectra of atomic oxygen. J. Chem. Phys. 39, 3159-3160 (1963).

40. Hagen, W.R. Dislocation strain broadening as s source of anisotropic linewidth and asymmetrical line shape on the electron paramagnetic resonance spectrum of metalloproteins and related systems. J. Magn. Reson. 44, 447-469 (1981). 


\section{METHODS}

\section{Reactor setup}

The plasma reactor $\left(\text { SunCell }{ }^{20}\right)^{20}$ comprised a reactor cell, a reaction cell chamber, a molten metal injector system with an electromagnetic pump driven by a DC power supply and a gallium reservoir that served as an electrode, a counter electrode, gas flow systems, bus bars to the electrodes, an ignition power source, and voltage, current, and temperature sensors. The reactor cell was a Type 347 stainless steel (SS) cylindrical tube measuring $7.3 \mathrm{~cm} \mathrm{ID,} 19.7 \mathrm{~cm}$ in height, and $0.635 \mathrm{~cm}$ thick with $3.17 \mathrm{~mm}$ thick boron nitride (99\%) liner to provide an electrical insulation barrier and a physical barrier to prevent the internal gallium inventory from alloying with the stainless steel at temperatures above $500{ }^{\circ} \mathrm{C}$. The cell was pressure leak checked at the shop following fabrication, and the high-vacuum integrity of the cell and gas and vacuum connections was confirmed by mass spectroscopy using a residual gas analyzer (Ametek Dycor Q100M). The cylindrical reaction cell chamber of about $50 \mathrm{ml}$ plasma volume was between the electrodes and confined internal to the $\mathrm{BN}$ liner. The molten metal injection system comprised $0.9 \mathrm{~kg}$ of molten gallium in the bottom of the rector that served as a reservoir of gallium and an

electrode, a Type 304 SS injection tube with a W injector nozzle submerged in the gallium by 0.7 $\mathrm{cm}$, and a DC electromagnetic pump. An electrical bus bar (W solid rod, $1 \mathrm{~cm} \mathrm{OD)} \mathrm{penetrated}$ the bottom of the reservoir through a Swagelok fitting (SS-10MO-1-6W) and was submerged in the gallium by at least $2.54 \mathrm{~cm}$ such that at least one of the injector nozzle and the molten gallium served as an electrode. The opposing counter electrode oriented along the negative $\mathrm{z}$ (vertical) axis (Extended Data Fig 5) received injected gallium from the injector nozzle to create an electrical connection between the two electrodes. The counter electrode comprised a feed through (solid sealing technology, part number FA10775) in a flange (Kurt Lesker 4.5 inch CF flange) sealed at the top of the reaction cell chamber by a gasket (silver plated $\mathrm{Cu}$ ), a W bus bar $1.37 \mathrm{~cm}$ diameter with male threads on each end to screw-in collect to a $2.54 \mathrm{~cm}$ diameter copper bus bar on the internal side of the feed through on the top end and the $\mathrm{W}$ electrode on the opposite end. The bus bar was covered by an electrically insulating fused quartz sheath (Technical Glass Products, $2.7 \mathrm{~cm}$ ID x $5.1 \mathrm{~cm}$ long) sealed with an inner quartz collar that penetrated into the feed through and was further sealed with an alumina-based cement (Resbond 989) at the feed-through end. The counter electrode, a concave refractory metal electrode (W 3.8 
$\mathrm{cm}$ OD, $1.37 \mathrm{~cm}$ height, with a concavity of $2 \mathrm{~cm}$ at the apex), screwed onto a threaded end of the $\mathrm{W}$ bus bar and pressed the quartz sheath against the top flange at the opposite end of the sheath.

\section{Production of reactants}

Reactants comprising nascent $\mathrm{H}_{2} \mathrm{O}$ catalyst and a source of atomic hydrogen were provided by separately controlled $\mathrm{H}_{2}$ (Praxair UHP, 99.999\%) and 8\% $\mathrm{O}_{2}$ (Praxair industrial grade) flows using two mass flow controllers (MKS Model PR4000-F2V1N with MKS Model 1179A53CR1BVS for 2500 SCCM of $\mathrm{H}_{2}$ flow and MKS Model M100B12R1BB for 200 SCCM of $\mathrm{O}_{2}$ flow) that were each calibrated with a rotameter (Dwyer Instruments VA10423, accurate to $+/-2 \%)$. The gases were mixed in an oxyhydrogen torch and flowed through a recombiner chamber comprising $1 \mathrm{~g}$ of a granular platinum catalyst ( $10 \% \mathrm{Pt} / \mathrm{Al}_{2} \mathrm{O}_{3}$ beads from Alfa Aesar) heated to greater than $90{ }^{\circ} \mathrm{C}$ by the $\mathrm{H}_{2}+1 / 2 \mathrm{O}_{2}$ recombination reaction before flowing into the reaction cell chamber using Swagelok fittings (SS-400-6-4W). The ignition system comprised either a switch-mode rectifier or a capacitor bank that supplied high-current DC electrical power sufficient to cause the reactants to react to form plasma. The current was measured with a Hall sensor and the voltage was measured with a PicoScope. The temperature of the molten gallium reservoir was measured using $2 \mathrm{~K}$-Type ungrounded thermocouples rated to $1335{ }^{\circ} \mathrm{C}$. The thermocouples penetrated the side section of the reaction cell chamber through Swagelok fittings (SS-200-6-2W) extended about $1 \mathrm{~cm}$ into the gallium.

Prior to the start of the reaction, the cell was connected to a scroll vacuum pump (Anest Iwata Model ISP-250) by a $2.54 \mathrm{~cm}$ OD stainless steel vacuum line with an intervening liquid nitrogen cryotrap. All unwanted gases were removed down to a pressure of approximately 40 mTorr (MKS Model 626A11TBE 10 Torr gauge and MKS Model 626A13TEE 1000 Torr gauge). During operation with flowing gas reactants, the pressure was maintained under 5 Torr. Prior to operation, the pressure gauges were verified for accuracy within $+/-1 \%$ using the same unit that was vendor calibrated.

\section{Reaction control}

The reaction within the cell was maintained using two separate electrical systems: an electromagnetic (EM) pump system to complete the circuit between the two electrodes within the cell, and an ignition system to supply electrical input energy to initiate the reaction. The EM 
pump was powered by a programmable DC power supply (Model: Matsusada Precision REK101200) set to current control mode wherein the current output directly controlled the flow rate of liquid Ga through the pump tube assembly. During typical operation, the Ga flow rate was measured to be approximately $40 \mathrm{~cm}^{3} / \mathrm{sec}$ wherein the voltage and current across the EM pump was about $0.1 \mathrm{~V}$ and $200 \mathrm{~A}$, respectively. The ignition circuit was powered by a LabVIEWcontrolled (National Instruments) switch-mode rectifier (Model: American CRS Q500 IP32) rated to a maximum of 50V/1500A. The negative and positive terminals of the rectifier were connected to a solid tungsten (W) rod anode and a liquid Ga cathode, respectively. The excess power was observed to be strongly dependent on the applied current. So, tests were also performed using an initial and peak current in the range of 3000-6000 A that was supplied by a capacitor bank charged to $48 \mathrm{~V}$ by the switch-mode rectifier. The capacitor bank comprised either four or eight Maxwell Technologies modules (Model BMOD0165 P048 C01) connected in parallel. Each module was rated to $48 \mathrm{~V}$ with a capacitance of $165 \mathrm{~F}$ wherein four or eight modules in parallel increased the capacitance to $660 \mathrm{~F}$ or $1320 \mathrm{~F}$, respectively. For the ignition circuit, the electrical response was recorded on a high sampling rate and high-resolution oscilloscope (Model: PicoScope 5000 Series) using a voltage differential probe (Model: PicoTech TA041, $\pm 70 \mathrm{~V}$ ) and a DC Hall effect sensor (Model: GMW CPCO-4000-77-BP10, \pm 4 kA). The Hall sensor was redundantly calibrated with three Matsusada DC power supplies (Model: Matsusada Precision REK10-1200) that were current calibrated by the manufacturer. The voltage probe was calibrated using a standardized voltage source (Model: Agilent E3631 A +/- $0.01 \mathrm{~V}$ ). The independently validated power developed was in the range of $200,000 \mathrm{~W}-340,000 \mathrm{~W}^{20-27}$.

\section{Product processing}

Either water or additional oxygen was flowed into the reaction cell chamber to form gallium oxide to entrap $\mathrm{H}_{2}(1 / 4)$ gas formed in the cell wherein the production of the $\mathrm{H}_{2}(1 / 4)$ gas was confirmed by gas chromatography following cryogenic collection as well as thermal release of gas from gallium-oxide trapped $\mathrm{H}_{2}(1 / 4)$ product $^{20}$. Gallium oxide material was collected from a hydrino reaction run in the SunCell@, and the gallium oxide material (50 g) was dissolved in $4 \mathrm{M}$ $\mathrm{KOH}$ solution $(500 \mathrm{ml})$. After $0.5-1$ hour, the solution was filtered to remove any insoluble solid phases. A white polymeric material began to nucleate from the clear filtrate after 24 hours. Using a Buchner funnel, side-arm flask, and filter paper (Whatman ${ }^{\mathrm{TM}}$, Grade 50, 09-865C), the 
ultrafine precipitate was suction filtered from the solution. The filtered compound was carefully removed from the filter paper with a spatula without contacting the filter paper. To wash the recovered compound, it was suspended in deionized (DI) water, and filtered out a second time using the prior procedure while applying additional DI water while filtering. The compound was dried in air at $60-80{ }^{\circ} \mathrm{C}$ for 12 hours. XRD showed that the resulting white polymeric material comprised $\mathrm{GaOOH}$ with an average particle size of $111 \mathrm{~nm}$.

\section{Product analysis}

Scanning electron microscopy (SEM) and energy dispersive X-ray spectroscopy (EDS) performed on polycrystalline powder samples mounted on a grid using an XL30 FEG-SEM equipped with an EVEX EDS, with an in-situ Tensile Stage, and a Gatan MiniCL imaging system. The lens magnification is $800 \mathrm{x}$ and secondary electrons are detected with resolution of 2 $\mathrm{nm}$. Electrons from the gun were accelerated at $10 \mathrm{kV}$ with working distance between the gun and sample adjusted to $24.3 \mathrm{~mm}$ within the low-pressure chamber.

Rutherford backscattering spectrometry (RBS) was performed on polycrystalline powder samples by EAG Laboratories, Sunnyvale CA using a NEC Pelletron, Model 3SDH particle accelerator with $\mathrm{He}++$ ions having an energy of $2.275 \mathrm{MeV}$, a normal detector angle of $160^{\circ}$, and grazing detector angle of $\sim 100^{\circ}$. The crystal channeling rotating random (CC RR) analysis mode was used to process the data.

Time-of-flight secondary ion mass spectrometry (ToF-SIMS) was performed on powder samples sprinkled onto the surface of a double-sided adhesive tape using a Physical Electronics nanoTOF TRIFT V ToF-SIMS instrument. The primary ion source was ${ }^{69} \mathrm{Ga}^{+}$at $30 \mathrm{kV}, 1 \mathrm{nA} \mathrm{DC}$, and bunched to $1 \mathrm{~ns}$. The scan parameters were a scan area of $250 \mu \mathrm{m} \times 250 \mu \mathrm{m}$, a mass range of 0-1850 amu), and a post acceleration voltage of $5000 \mathrm{~V}$ with acquisition modes of positive and negative. Charge compensation was applied to the electron gun and gas gun during position acquisition and applied to the electron gun only during negative acquisition. The aperture size was $100 \mathrm{~m}$. In order to remove surface contaminants and expose a fresh surface, the samples were sputter-cleaned for 800 seconds prior to data collection using a $1 \mathrm{~mm} \times 1 \mathrm{~mm}$ raster of the ${ }^{69} \mathrm{Ga}^{+}$gun at a total ion dose of $10^{15}$ ions $/ \mathrm{cm}^{2}$. Both the positive and negative spectra were obtained with a total ion dose of $10^{12}$ ions $/ \mathrm{cm}^{2}$. 
Transmission electron microscopy (TEM), selected area electron diffraction pattern (SAED), and scanning transmission electron microscopy (STEM) were recorded on solid samples, each mounted on a copper grid using a Thermo Scientific Talos F200X Scanning/Transmission Electron Microscope (S/TEM) equipped with an X-FEG source and a super-X energy dispersive spectrometer (super-X EDS). The system was operated at $200 \mathrm{kV}$, and the electron source brightness was $1.8 \times 10^{9} \mathrm{~A} / \mathrm{cm}^{2}$ srad at $200 \mathrm{kV}$ which provided a point resolution of $0.12 \mathrm{~nm}$ for TEM and an information resolution of $0.16 \mathrm{~nm}$ for STEM. High vacuum of less than $10^{-7}$ Torr and high-resolution electron tension were maintained at $200 \mathrm{kV}$.

X-ray diffraction (XRD) was performed on powder samples ground with a mortar and pestle prior to testing. The samples were then loaded into standard sample holders and placed into a Panalytical X'pert diffractometer using $\mathrm{Cu}$ radiation at $45 \mathrm{kV} / 40 \mathrm{~mA}$, and the scans were run over the range of $6^{\circ}$ to $80^{\circ}$ with a step size of $0.0167^{\circ}$ and an accumulated counting time of 250 seconds per step. Once the patterns had been collected, the crystalline phases were identified with the aid of the Powder Diffraction File published by the International Centre for Diffraction Data or the Inorganic Crystal Structure Database.

\section{EPR spectroscopy}

X-band spectra were recorded at 9.4-9.9 GHz with a Bruker EMX-plus spectrometer using the high-sensitivity ER4119HS resonator. The microwave frequency was monitored with a $20 \mathrm{GHz}$ Hewlett Packard 5350B frequency counter. Q-band data were taken at ca $35 \mathrm{GHz}$ with a Varian E-line spectrometer with the recorder $\mathrm{y}$-axis amplitude and the $\mathrm{x}$-axis micro switches connected to a NI 6001 data acquisition interface (National Instruments) for $20 \mathrm{kS} / \mathrm{s}$ digital storage using a LabVIEW program. A WR-28 waveguide 566-series cross guide directional coupler combined with a 410-series waveguide to coax transition (Millimeter Wave Products) were built into the main microwave path of the Q-band bridge to monitor the frequency with a $46 \mathrm{GHz}$ Hewlett Packard 5352B frequency counter. Frequency counters were connected to PC's via USB IEEE 488 GPIB interfaces (National Instruments) to log frequency drift over extended data-collection times with a LabVIEW program for later off-line normalization. For Q-band the static magnetic field value was permanently monitored in repetitive sans with a F55 field meter (Magnet-Physik) equipped with an extra-long axial probe, and data were logged with a LabVIEW program. 
Individual scans for signal averaging were normalized for drifts in field and/or frequency with a LabVIEW program before averaging.

\section{EPR data analysis}

The Bruker spectrometer can be set to automatically collect $2 \mathrm{D}$ data sets for varying microwave power intensity (that is, EPR amplitude versus magnetic field and microwave power), but the manufacturer's software lacks an option to analyze these data in terms of inhomogeneous broadening. Therefore, a LabVIEW program was written for non-linear Levenberg-Marquardt fitting to Portis' theory ${ }^{34}$.

EPR spectra taken at two different frequencies were compared in a LabVIEW program that afforded frequency normalization versus field scan shift normalization in order to separate frequency-dependent from frequency-independent spectral components.

To generate simulations of EPR spectra two programs were written in Intel FORTRAN with a GUI written in LabVIEW. The first program simply uses the field values predicted by hydrino theory (Supplementary Information) in combination with symmetric Gaussians for the individual $\mathrm{m}=1 / 2$ fluxon lines (standard deviation, $\sigma=130 \mathrm{mGauss}$ ), for their distribution $(\sigma=$ 1.30 Gauss), for the $\mathrm{m} \geq 1$ satellite spin-orbital fluxon lines ( $\sigma=124 \mathrm{mGauss})$, and for the broad underlying feature ( $\sigma=6.0$ Gauss) with relative amplitudes for the $\mathrm{m}=1 / 2$ spectrum, the $\mathrm{m} \geq 1$ spectrum, and the broad spectrum fitted as $100: 8: 19$. The second program is a classical spinHamiltonian simulator in which hyperfine interactions are taken to second-order perturbation of the electronic Zeeman interaction ${ }^{41,42}$.

Spin quantification ${ }^{43}$ was done with respect to an external standard of known $S=1 / 2$ concentration. For this purpose, the X-band spectrum of a powder of $0.5 \%$ (metal $/ \mathrm{metal}^{\text {) of }}{ }^{44}$ $\mathrm{Cu}$ (II) in $\mathrm{Zn}(\mathrm{II}) \mathrm{SO}_{4} \cdot 1 \mathrm{H}_{2} \mathrm{O}$ was taken, which has essentially the same density (weight/volume) as the hydrino-containing $\mathrm{Ga}(\mathrm{O}) \mathrm{OH}$ powder. 


\section{Data availability}

All data necessary to evaluate the claims of this paper are provided in the main manuscript and Supplementary information. Raw data files, instrument controlling code, data analysis code and simulation code are freely available upon request.

41. Pake, G.E. \& Estle, T.L. The physical principles of electron paramagnetic resonance $2^{\text {nd }}$ edn (W.A. Benjamin, 1973).

42. Hagen, W.R. EPR spectroscopy as a probe of metal centers in biological systems. Dalton Trans. 2006, 4415-4434.

43. Hagen, W.R. Biomolecular EPR spectroscopy (Taylor \& Francis 2009).

44. Hagen, W.R. Broadband tunable electron paramagnetic resonance spectroscopy of dilute metal complexes. J. Phys. Chem. 123, 6986-6995.

\section{Acknowledgements}

We are grateful to Dr Peter van Noorden for creating the liaison between the authors.

\section{Author contributions}

RLM developed the theory and supervised the production and analysis of the samples (with technical assistance by the authors of Supplementary information, ZD, JJ, RG, NSM, SM \& PS); WRH did the EPR experiments and wrote the dedicated software; WRH and RLM wrote the manuscript; RLM wrote the Supplementary information.

Competing interests The authors declare no competing interests.

\section{Additional information}

Correspondence and requests for materials should be addressed to WRH or RLM 


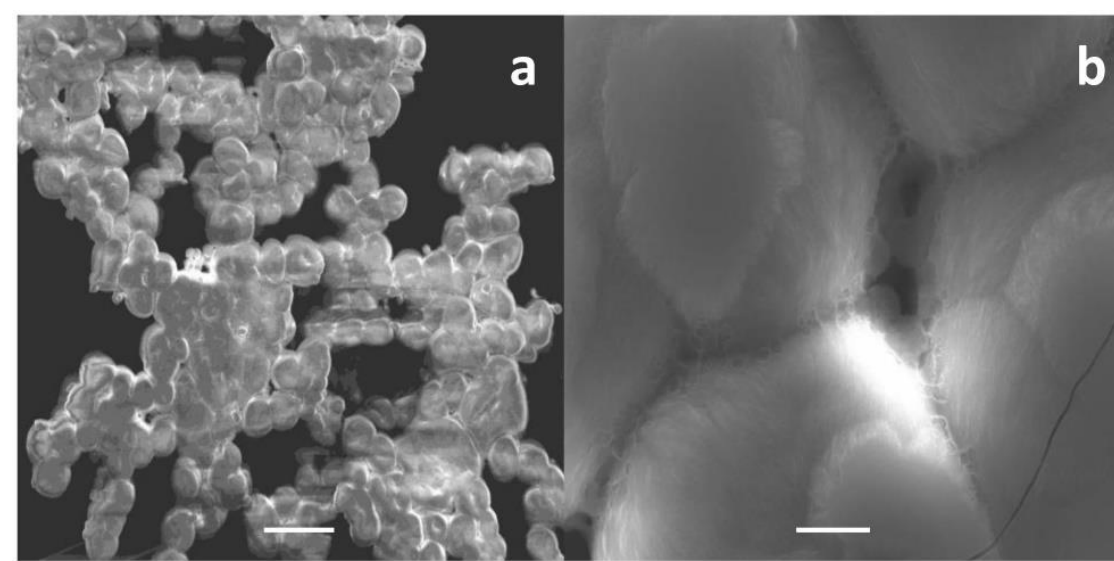

$20 \mu \mathrm{m}$

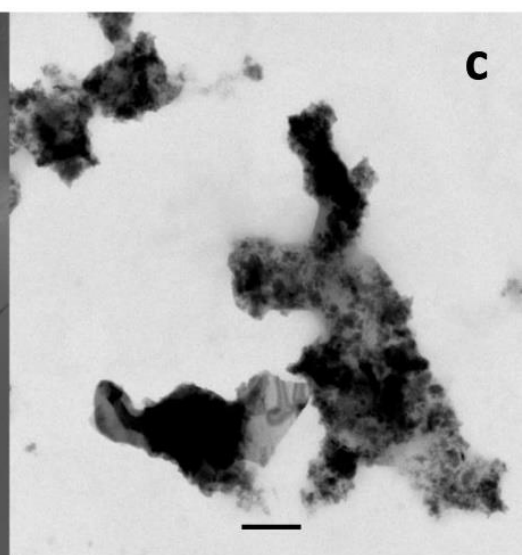

$500 \mathrm{~nm}$

Fig. 1 Scanning electron microscopy and transmission electron microscopy of $\mathbf{G a}(\mathrm{O}) \mathrm{OH}: \mathrm{H}_{2}(\mathbf{1} / 4)$. Trace a: SEM at $800 \times$ magnification showing chains of microspherical particles; trace b: SEM showing $5 \mu \mathrm{m}$ width of the particles, each comprising very fine fibers; trace c: TEM imaging of morphologically polymeric crystals of hexagonal structure (Extended Data Fig. 2), which were very sensitive to the TEM electron beam. Observed spherical particles have approximately $100 \mathrm{~nm}$ average diameter. 


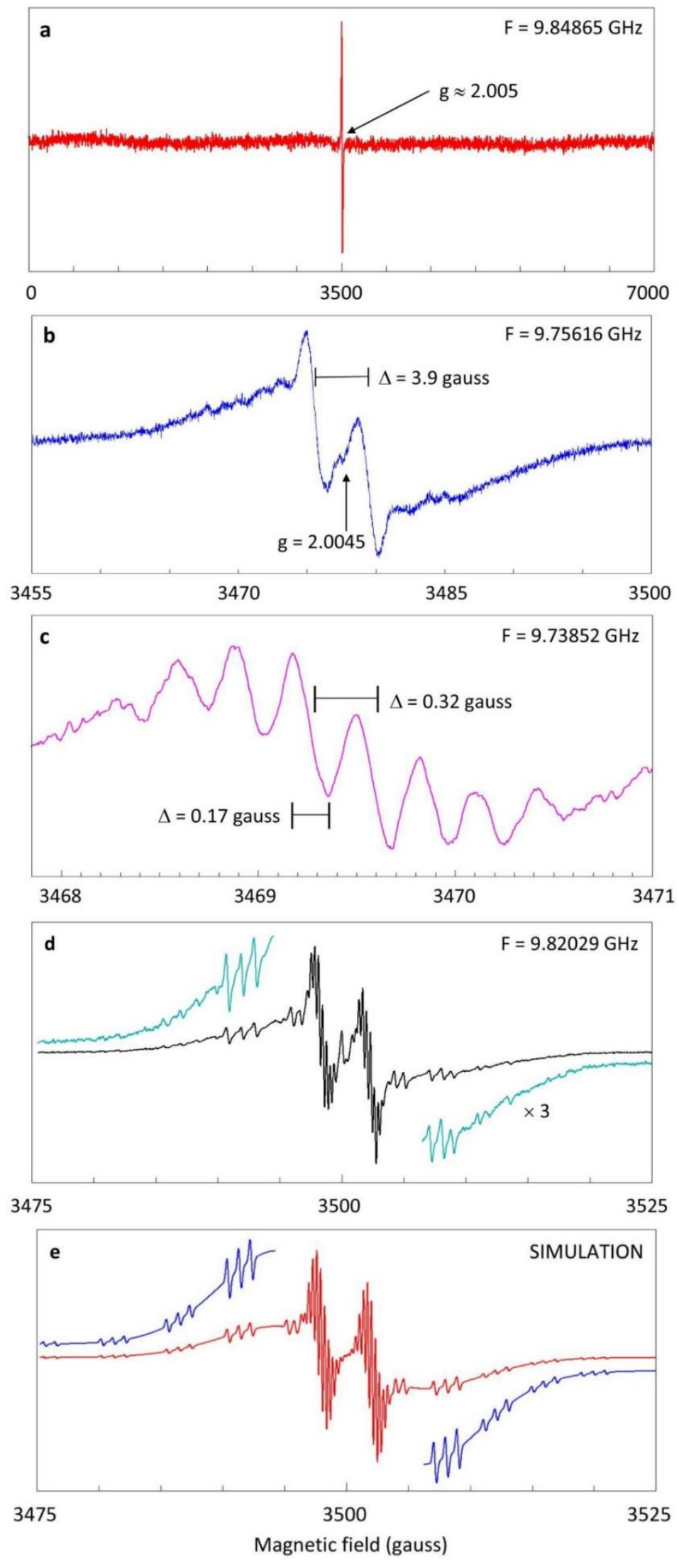

Fig. 2 EPR of postulated molecular hydrino $\mathrm{H}_{2}(1 / 4)$ caged in solid

$\mathrm{Ga}(\mathrm{O}) \mathrm{OH}$ polymer. Trace a: wide-scan overview spectrum showing a single feature only close to the free electron $g$ value. Trace b: zoom-in of the single feature in trace a shows two main lines of equal intensity, separated by circa 4 Gauss, and whose center is distinctly shifted from the free electron value to $\mathrm{g}$ $=2.0045$. Trace $\mathrm{c}$ : further zoom-in on one of the lines now recorded with a very small modulation amplitude reveals a fine structure of multiple lines with apparent peak-to-peak derivative line width of 0.17 Gauss and separated by circa 0.32 Gauss. Trace d: extensively averaged spectrum taken under conditions optimized for maximal signal-to-noise ratio at the expense of minor over-modulation and power saturation, exhibits a complex pattern of triplet satellite lines. Data collection times for traces a-d were 10, 16, 375, and $2400 \mathrm{~min}$, respectively. All spectra were taken at ambient temperature. Trace e is a simulation using field positions predicted by hydrino theory. Other experimental conditions and simulation parameters are given in the METHODS section. 

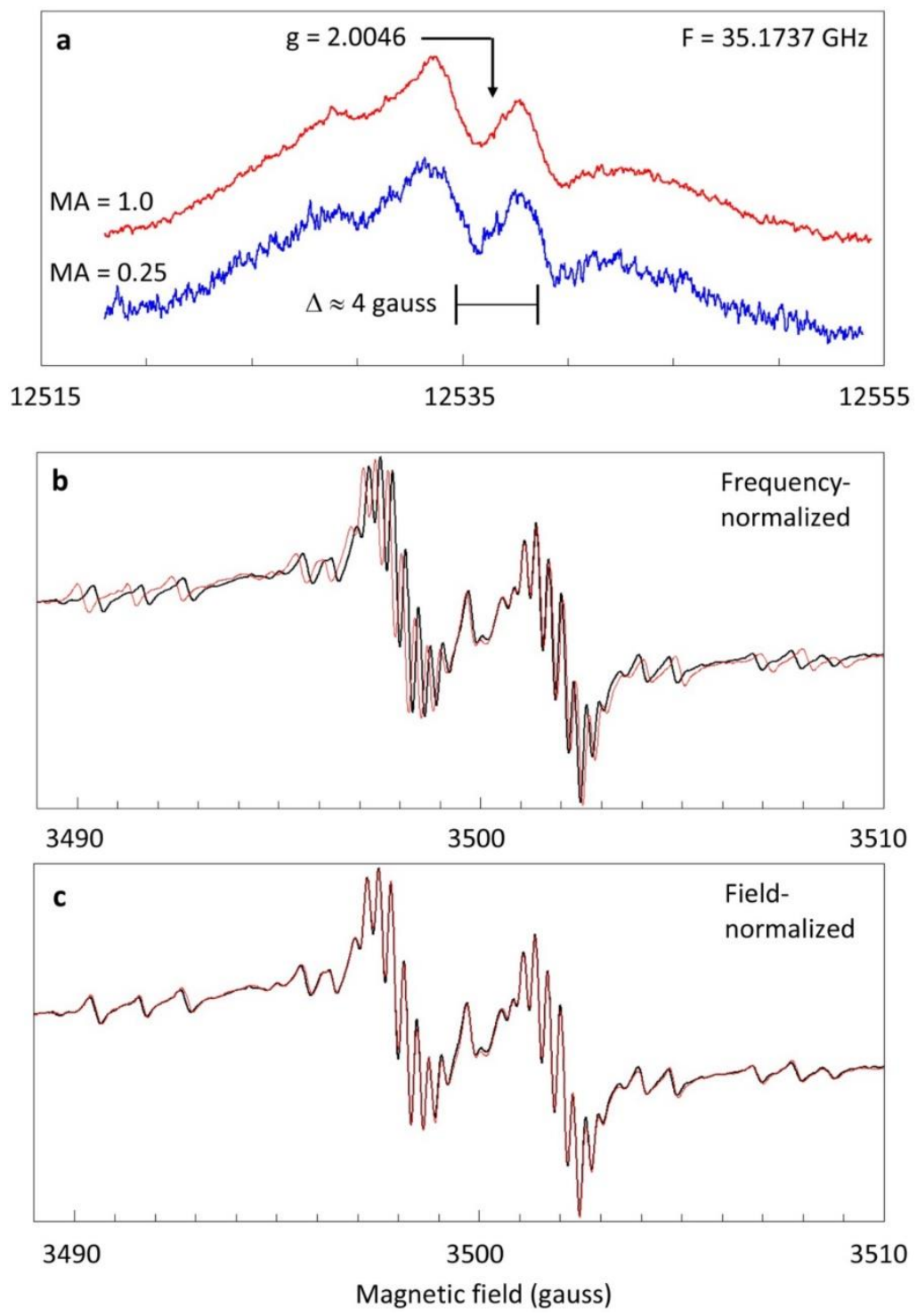

\section{Fig.3 Multi-frequency experiments as checks on consistency of the EPR interpretation.}

Trace a: extensively averaged Q-band spectra taken at two different modulation amplitudes of 1 Gauss (red) or 250 mGauss (blue). No fine structure is resolved in addition to the two main lines consistent with an inhomogeneous line width linear in the microwave frequency. The central $g$ value and the splitting between the two lines in field units are identical to those observed in X-band. Trace b: extensively averaged intra X-band experiment at two frequencies, $9.4629 \mathrm{GHz}$ (red) and $9.8209 \mathrm{GHz}$ (black). Each field point of the red spectrum is frequency transformed to that of the black spectrum where the overlay shows that only the center of the two main lines is a real $\mathrm{g}$ value. In trace $\mathrm{c}$ the red spectrum is shifted in its entirety to a higher field for maximal overlap with the black spectrum. Here the overlay proves that there is only a single real g value and that all other features are constant in the field. See METHODS for experimental conditions. 


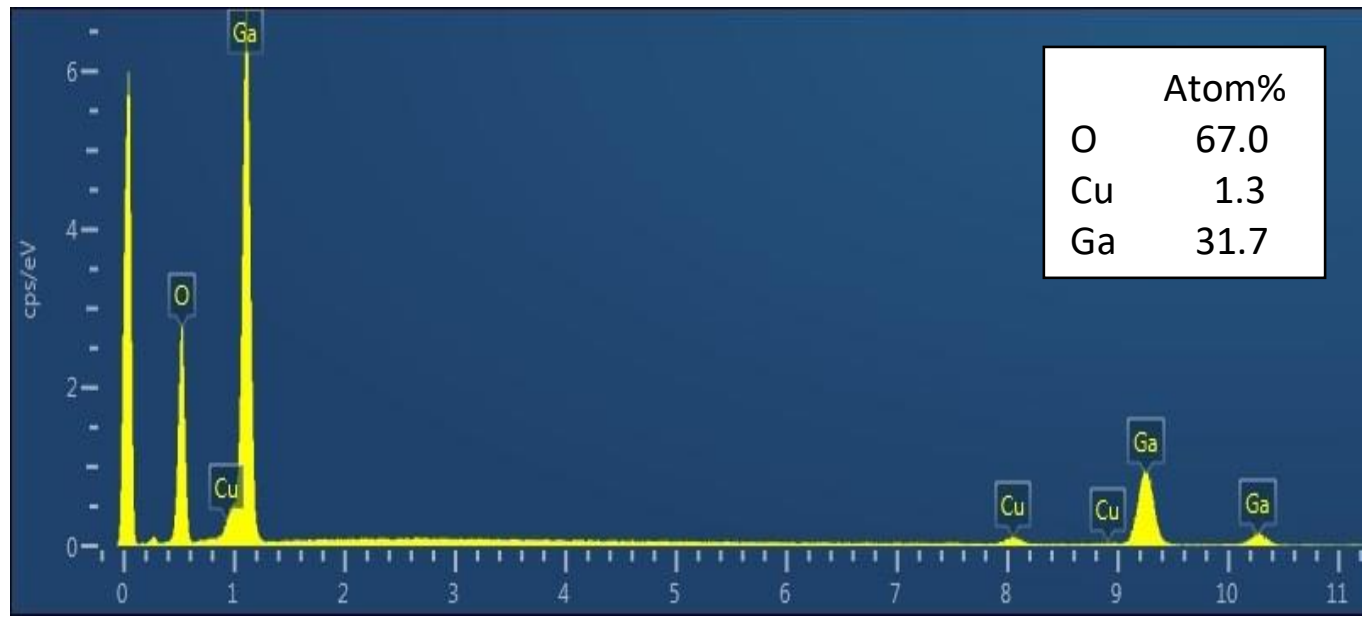

Extended Data Fig. 1 Energy dispersive X-ray spectroscopy of Ga(O)OH:H2(1/4). Only $\mathrm{Ga}$ and $\mathrm{O}$ are detected in a stoichiometry of $\mathrm{GaO}_{2.1}$. $\mathrm{Cu}$ signals are from the TEM grid. 

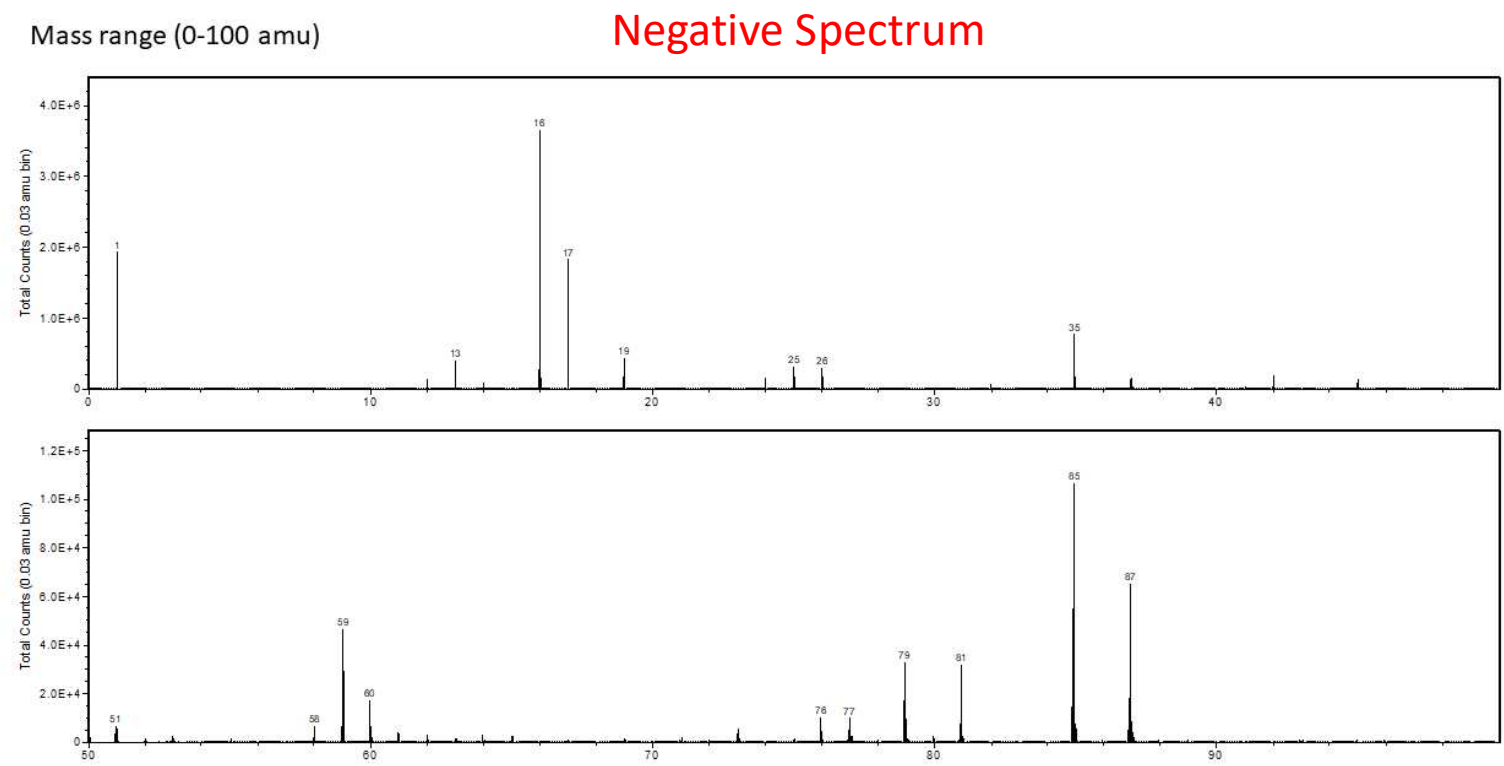

Mass range (0-100 amu)
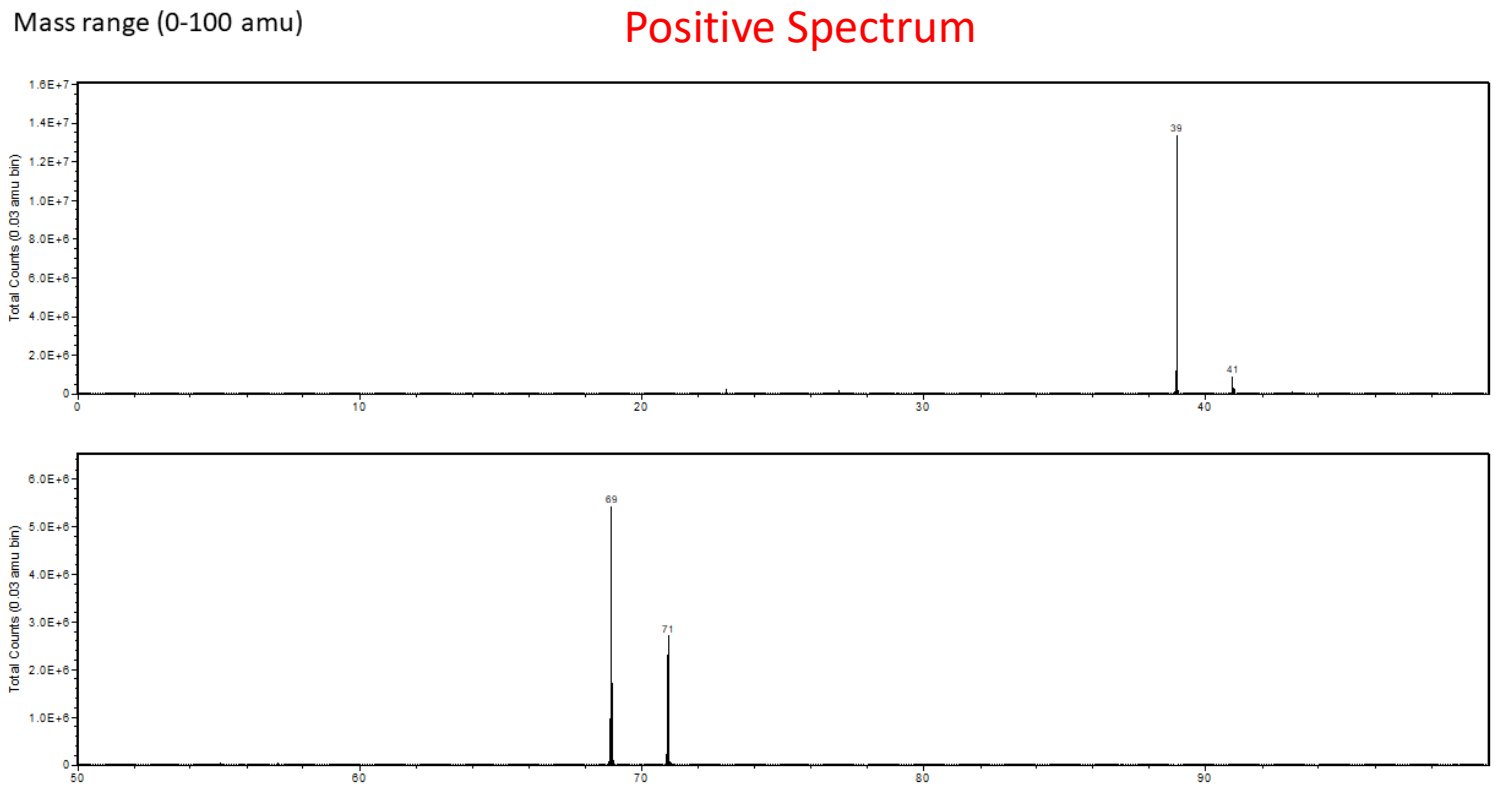

Extended Data Fig. 2 Ga ion time-of-flight secondary ion mass spectrometry of

$\mathbf{G a}(\mathbf{O}) \mathrm{OH}: \mathbf{H}_{2}(\mathbf{1 / 4})$. The negative spectrum shows hydride ion as a dominant ion fragment due to the stability of hydrino hydride ion. The result confirms the $\mathrm{H}$ content supporting the molecular hydrino component. No hydrocarbons above adventitious HC and no $\mathrm{N}$ containing fragments were present that could give rise to a radical EPR spectrum. In the positive spectrum only $\mathrm{K}$ and $\mathrm{Ga}$ were observed. No transition metals were present that could give rise to an EPR spectrum. 

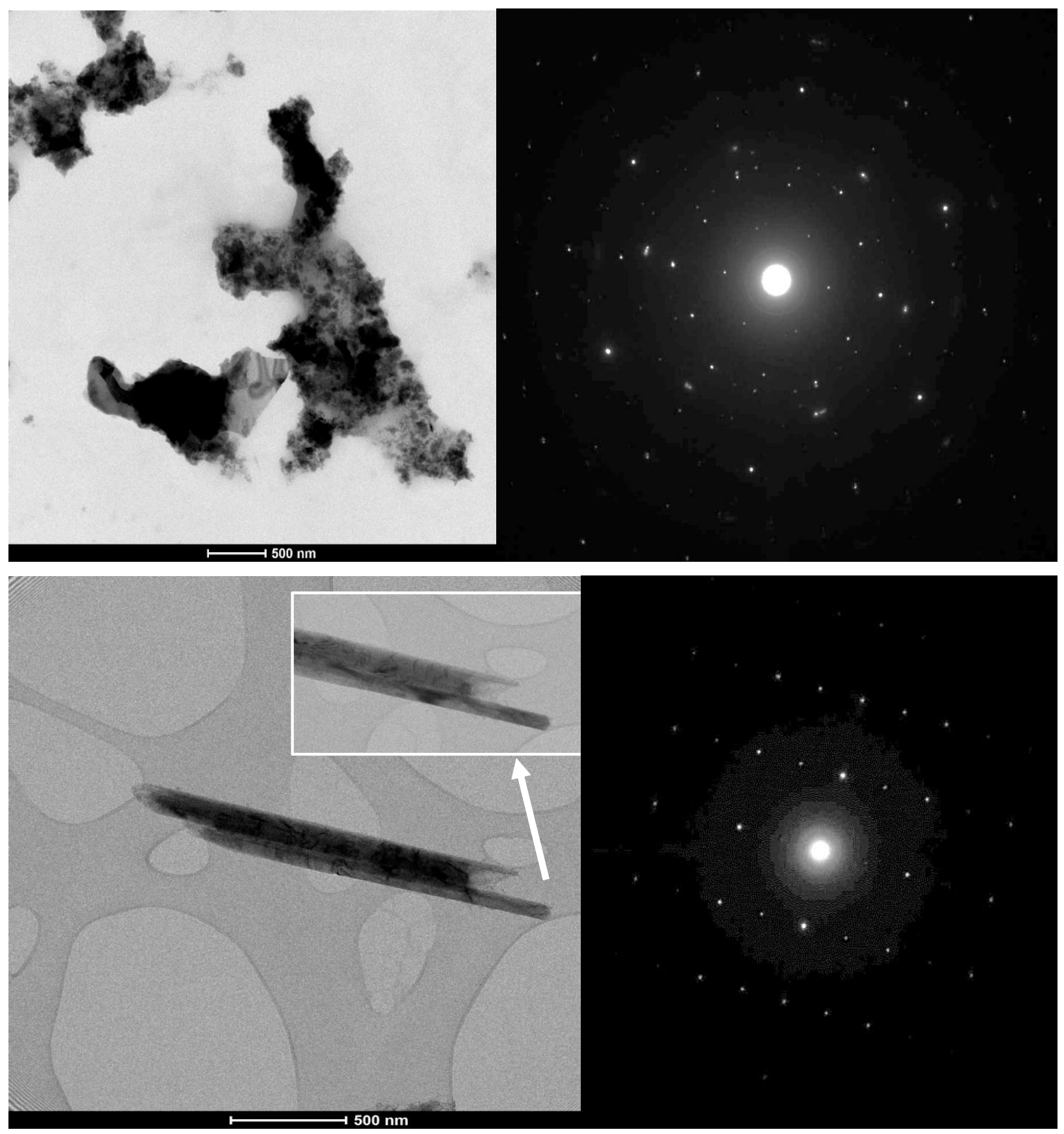

Extended Data Fig. 3 Transmission electron microscopy and selected area electron diffraction analysis for $\mathbf{G a}(\mathbf{O}) \mathbf{O H}: \mathbf{H}_{2}(\mathbf{1} / 4)$. The sample was observed to comprise two different morphological and crystalline forms of $\mathrm{Ga}(\mathrm{O}) \mathrm{OH}$ : polymeric crystals with hexagonal structure (upper panels) and rods with orthorhombic crystalline structure (lower panels). The polymeric crystals were very sensitive to the TEM electron beam, whereas the rods were not electron-beam sensitive. Properties of the rods correspond to literature values for $\mathrm{Ga}(\mathrm{O}) \mathrm{OH}$ that lacks gaseous molecular hydrino inclusion. 

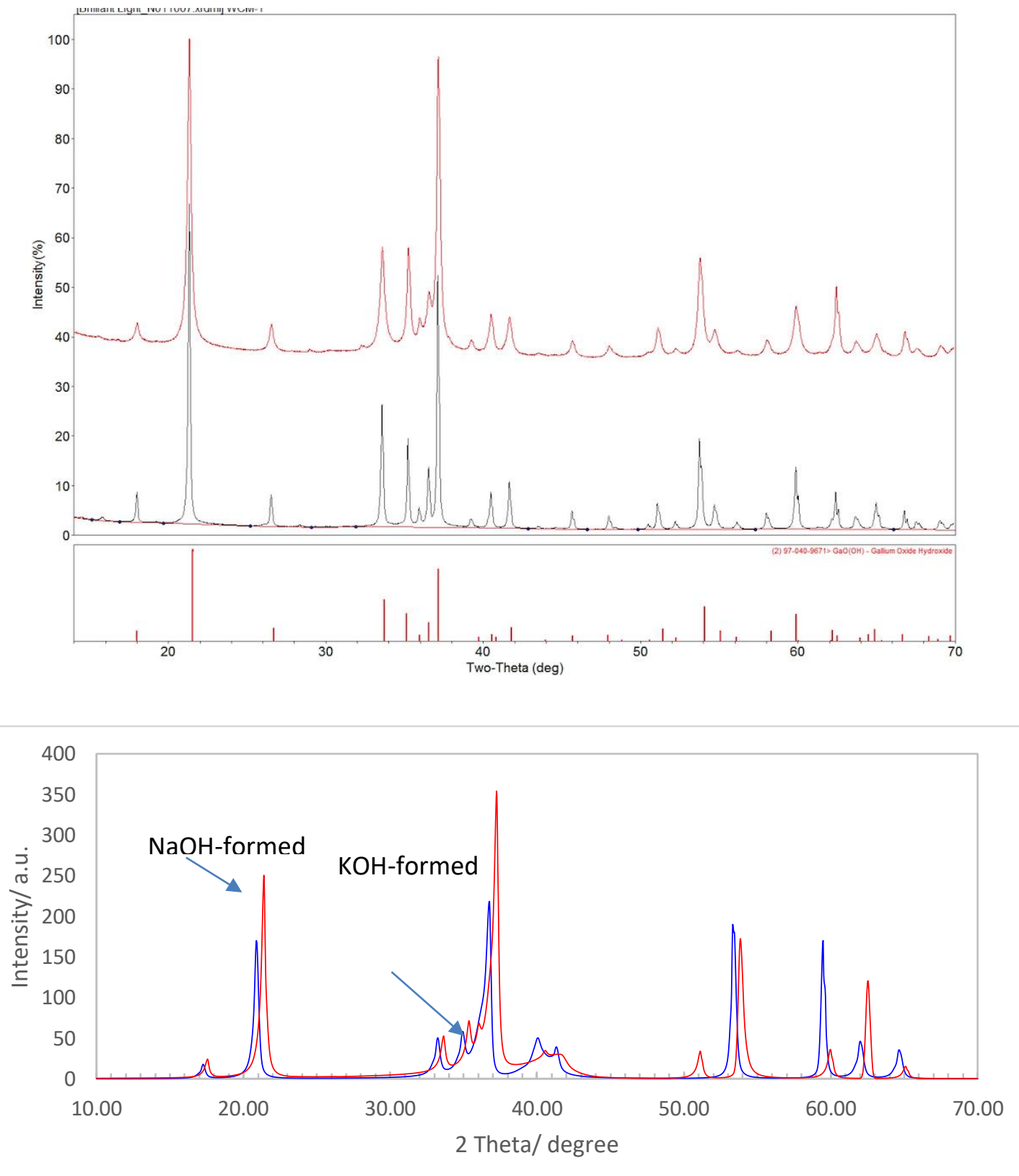

Extended Data Fig. 4 X-ray diffraction of $\mathbf{G a}(\mathrm{O}) \mathrm{OH}: \mathrm{H}_{2}(1 / 4)$. Samples were formed by dissolving $\mathrm{Ga}_{2} \mathrm{O}_{3}$ collected from a hydrino reaction run in $4 \mathrm{M}$ aqueous $\mathrm{KOH}$ or $4 \mathrm{M}$ $\mathrm{NaOH}$, allowing fibers to grow, and float to the surface where they were collected by filtration. The $\mathrm{KOH}$ pattern is shifted to lower $2 \theta$ relative to that of $\mathrm{NaOH}$ and both patterns are shifted relative to the standard pattern of $\mathrm{Ga}(\mathrm{O}) \mathrm{OH}$. 

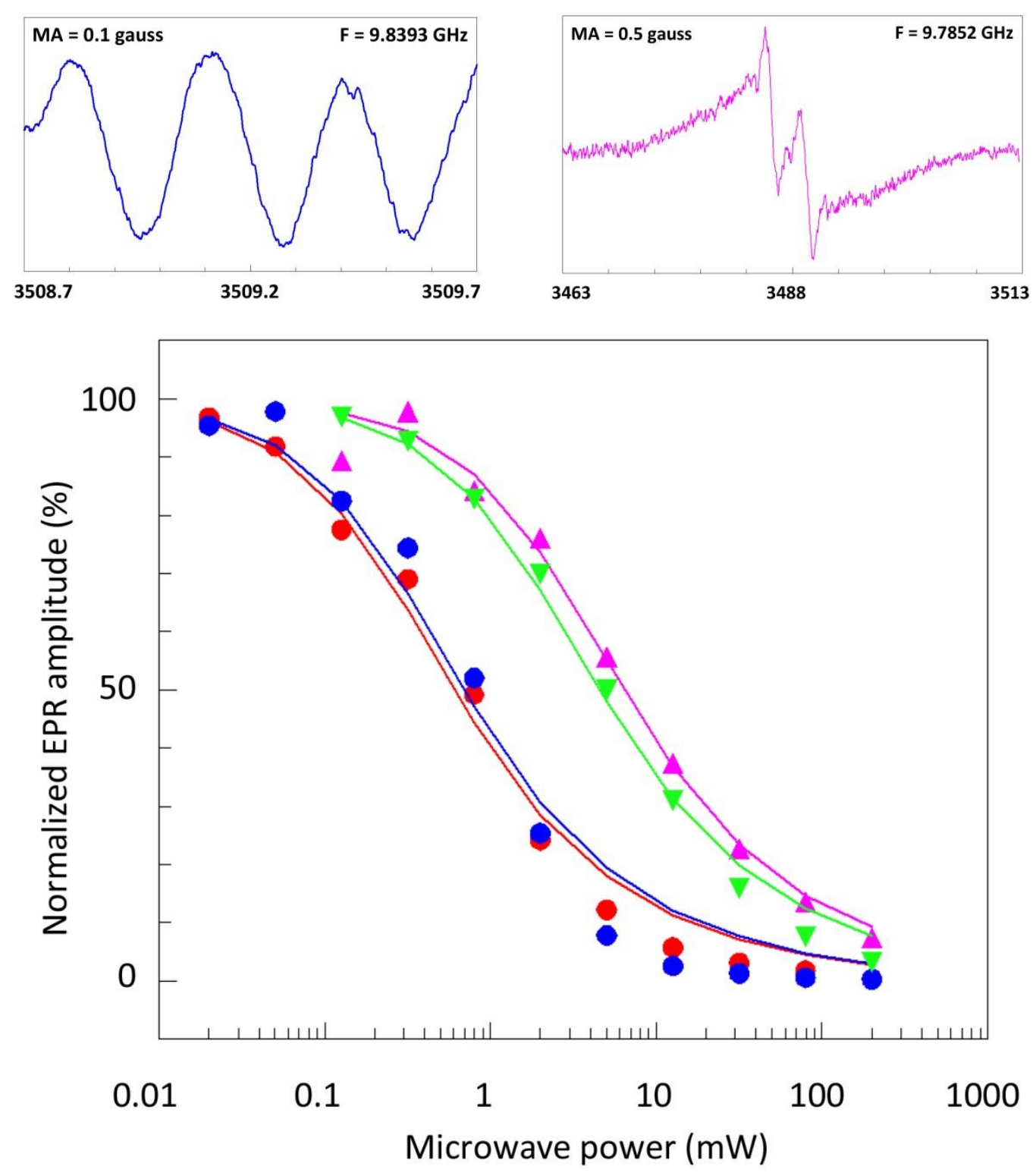

Extended Data Fig. 5 The power-saturation characteristics of the two main peaks and their fluxonal sub-peaks. Upper panels are example spectra taken at ambient temperature under proper modulation (modulation amplitude $=0.1$ Gauss; power $=0.13 \mathrm{~mW})$ or over-modulation $(\mathrm{MA}=0.5$ Gauss; power $=2 \mathrm{~mW}$ ). The lower panel gives power plots of the left peak (magenta) and right peak (green) as well as for fluxonal sub-peaks of the left peak (red) or the right peak (blue). The fit is according to Portis, case-3, that is for inhomogeneous broadening with relatively slow $\mathrm{T}_{1}$ relaxation time $^{34}$. The data indicate full inhomogeneous broadening, therefore, the observed line width should be linear in the frequency and the homogeneous line width should be significantly less than the sharpest observed line (155 mGauss). 

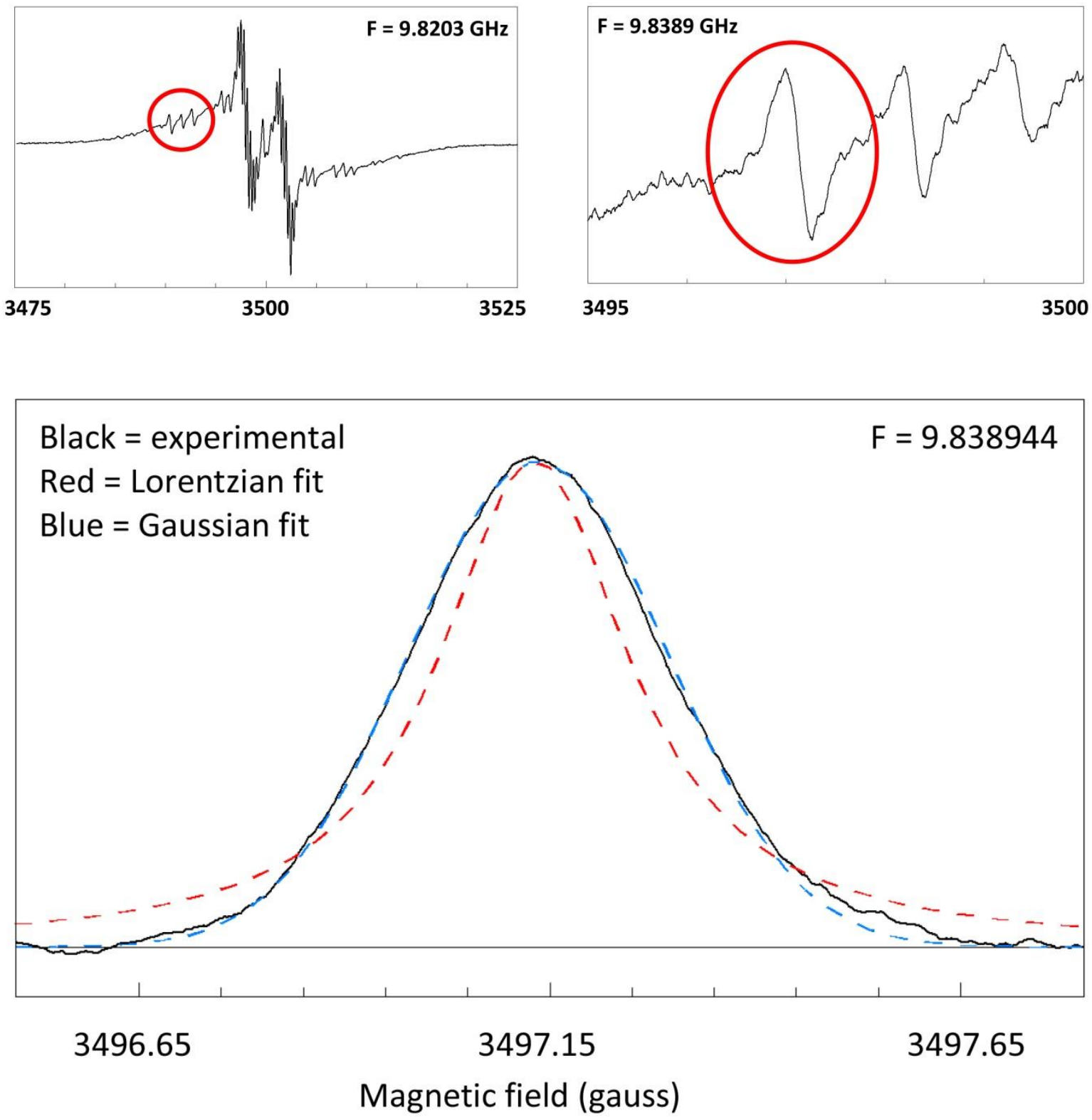

Extended Data Fig. 6 The line shape is Gaussian. The upper panels indicate the origin of the resonance line, which in the lower panel has been integrated and fitted to different line shapes. The optimal Lorentzian fit has 0.1405 Gauss half-width-at-halfheight and coefficient of determination $\mathrm{R}^{2}=0.934$; the optimal Gaussian fit has 0.1578 gauss standard deviation and $\mathrm{R}^{2}=0.998$. The observed purely Gaussian line shape is taken to imply linearity of the line width with microwave frequency ${ }^{40}$. 


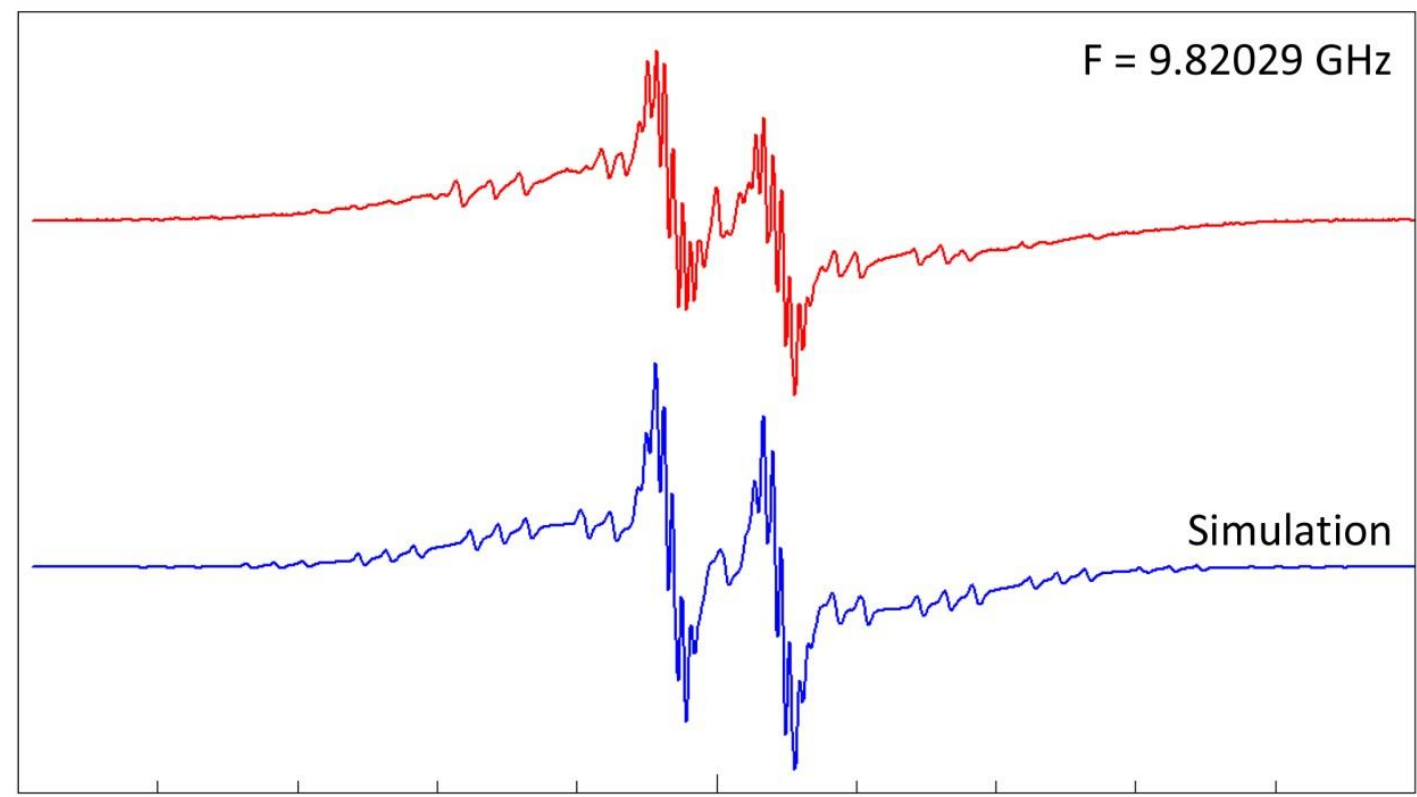

3475

3500

3525

Magnetic field (gauss)

Extended Data Fig. 7 Alternative interpretation of the EPR spectrum assuming a complex radical pattern. The simulation assumes two radicals each with an isotropic $\mathrm{g}$ value of 2.045 and in a 1:0.08 concentration ratio. The first radical gives rise to the two main lines by means of isotropic hyperfine coupling $\mathrm{A}_{\text {iso }}=0.3$ Gauss to a single $\mathrm{I}$ $=3 / 2$ nucleus, an $\mathrm{A}_{\text {iso }}=0.3$ Gauss coupling to four $\mathrm{I}=0.5$ nuclei and an $\mathrm{A}_{\text {iso }}=3.88$ Gauss coupling to another $\mathrm{I}=0.5$ nucleus. Alternatively, the latter splitting can also be generated by two identical radials at a mutual distance of circa $11 \AA$ in isotropic dipolar interaction equal to 3.88 Gauss. The second radical is subject to hyperfine coupling with an $\mathrm{I}=2$ nucleus with $\mathrm{A}_{\text {iso }}=4$ Gauss, three $\mathrm{I}=1$ nuclei with $\mathrm{A}_{\text {iso }}=4$ Gauss and another $\mathrm{I}=1$ nucleus with $\mathrm{A}_{\mathrm{iso}}=1$ Gauss. All lines have a half-width-athalf-height of $\mathrm{W}=160 \mathrm{mGauss}$. A broad underlying signal is also simulated as a single line with $\mathrm{g}=2.0045$ and linewidth $\mathrm{W}=6$ Gauss. In view of the number of fitting parameters required, the fit may not be unique; it is presented to illustrate the necessity to assume a very complex - and therefore highly unlikely - radical structure to reproduce the experimental spectrum under the assumption of a conventional spin Hamiltonian encompassing isotropic Zeeman interaction plus hyperfine interaction with multiple nuclei. 


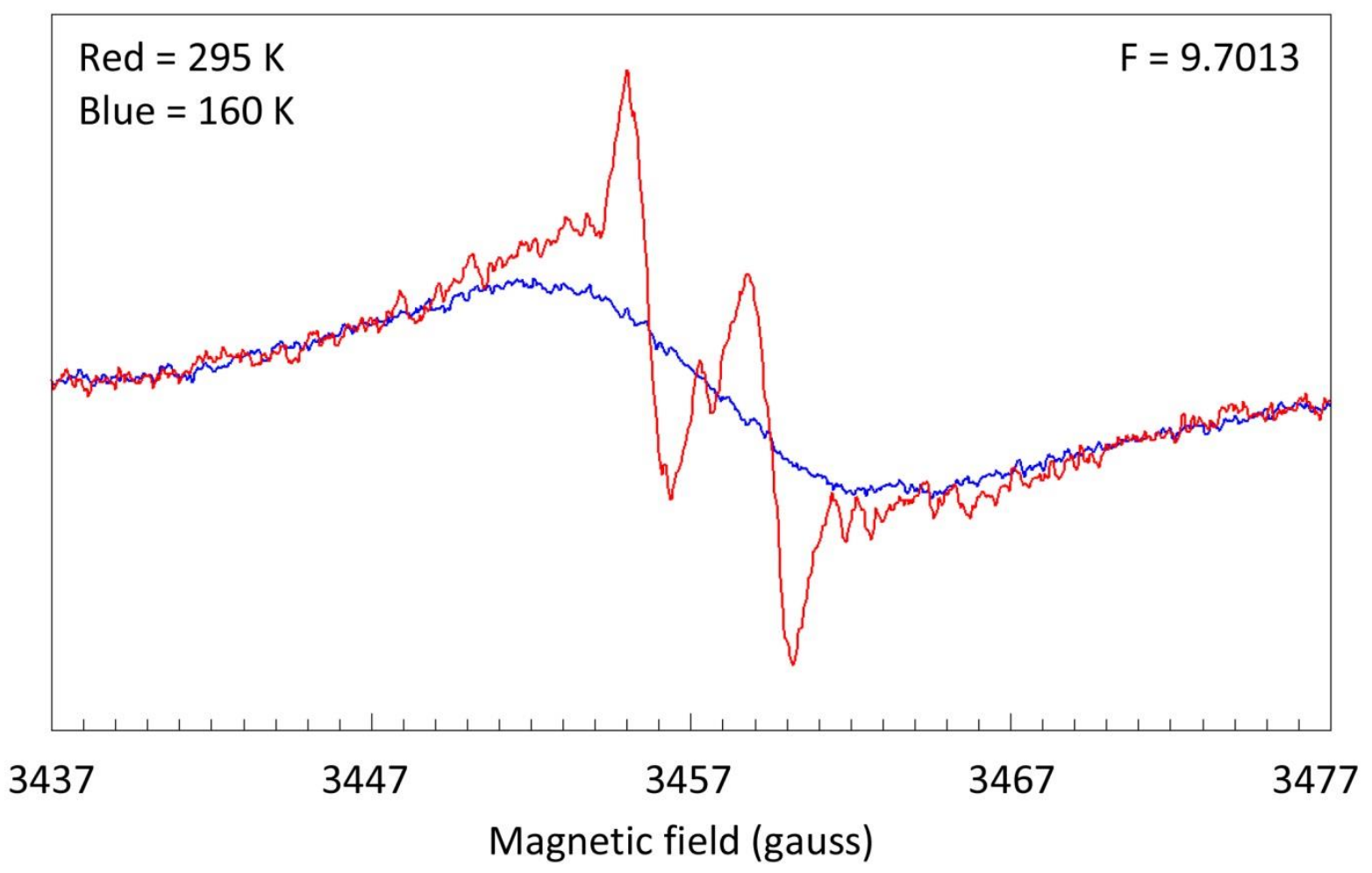

Extended Data Fig. 8 Differential temperature dependence of the putative molecular hydrino signal and a broad underlying signal. Upon lowering the measuring temperature from ambient to $160 \mathrm{~K}$ the hydrino spectrum completely disappears by saturation. The underlying broad signal is not affected clearly indicating that it is not part of the hydrino fine-structure spectrum. Its origin is yet to be identified, but is likely due to the orthorhombic phase of $\mathrm{GaOOH}$. The blue spectrum has been transformed to the frequency of the red spectrum; its amplitude has been arbitrarily adjusted for easy visual comparison. EPR conditions: frequency, $9.4011 \mathrm{GHz}$ (blue) or $9.7013 \mathrm{GHz}$ (red); microwave power, 2 $\mathrm{mW}$ (blue) or $0.8 \mathrm{~mW}$ (red); modulation frequency, $100 \mathrm{kHz}$; modulation amplitude, 0.5 Gauss. 


\section{Figures}

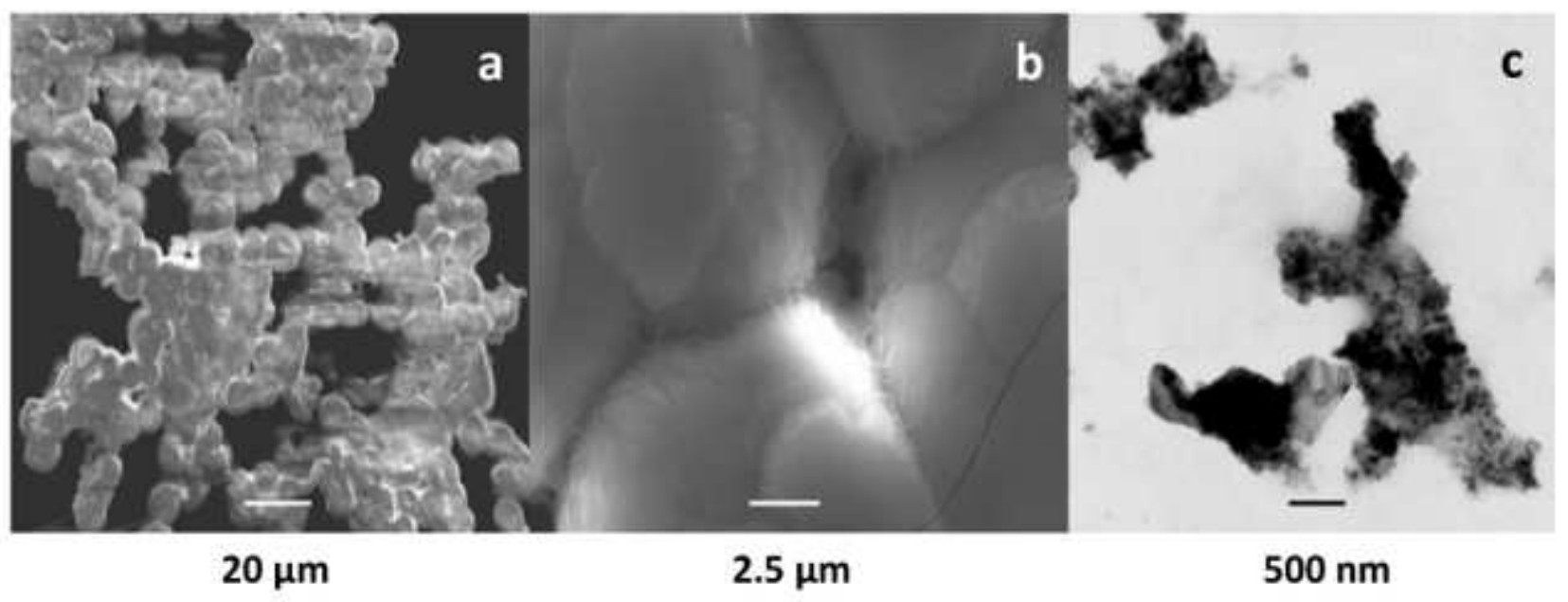

\section{Figure 1}

Scanning electron microscopy and transmission electron microscopy of $\mathrm{Ga}(0) \mathrm{OH}: \mathrm{H} 2(1 / 4)$. Trace a: SEM at $800 x$ magnification showing chains of microspherical particles; trace b: SEM showing $5 \mu \mathrm{m}$ width of the particles, each comprising very fine fibers; trace c: TEM imaging of morphologically polymeric crystals of hexagonal structure (Extended Data Fig. 2), which were very sensitive to the TEM electron beam. Observed spherical particles have approximately $100 \mathrm{~nm}$ average diameter. 

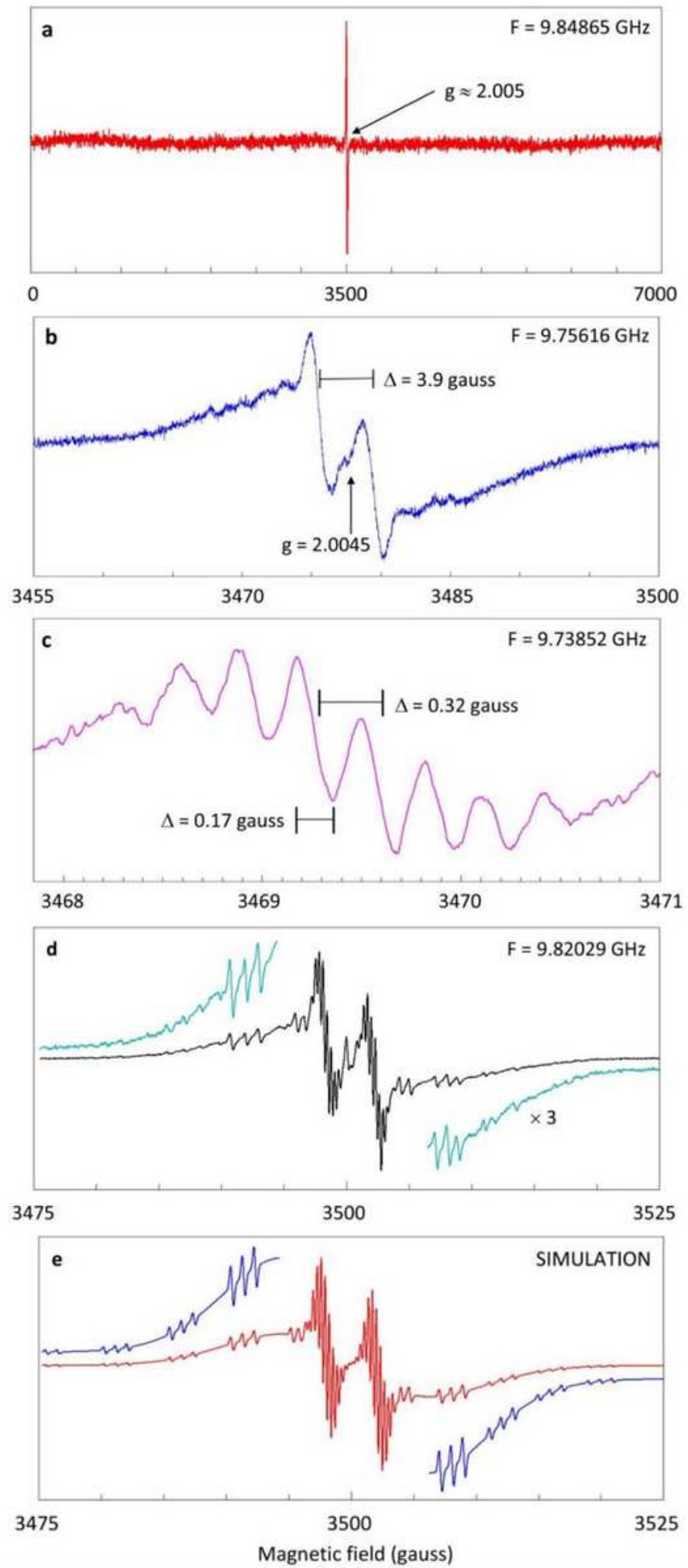

Figure 2

EPR of postulated molecular hydrino $\mathrm{H} 2(1 / 4)$ caged in solid $\mathrm{Ga}(0) \mathrm{OH}$ polymer. Trace a: wide-scan overview spectrum showing a single feature only close to the free electron g value. Trace b: zoom-in of the single feature in trace a shows two main lines of equal intensity, separated by circa 4 Gauss, and whose center is distinctly shifted from the free electron value to $g=2.0045$. Trace $\mathrm{c}$ : further zoom-in on one of the lines now recorded with a very small modulation amplitude reveals a fine structure of multiple 
lines with apparent peak-to-peak derivative line width of 0.17 Gauss and separated by circa 0.32 Gauss. Trace d: extensively averaged spectrum taken under conditions optimized for maximal signal-to-noise ratio at the expense of minor over-modulation and power saturation, exhibits a complex pattern of triplet satellite lines. Data collection times for traces a-d were 10, 16, 375, and $2400 \mathrm{~min}$, respectively. All spectra were taken at ambient temperature. Trace $\mathrm{e}$ is a simulation using field positions predicted by hydrino theory. Other experimental conditions and simulation parameters are given in the METHODS section.
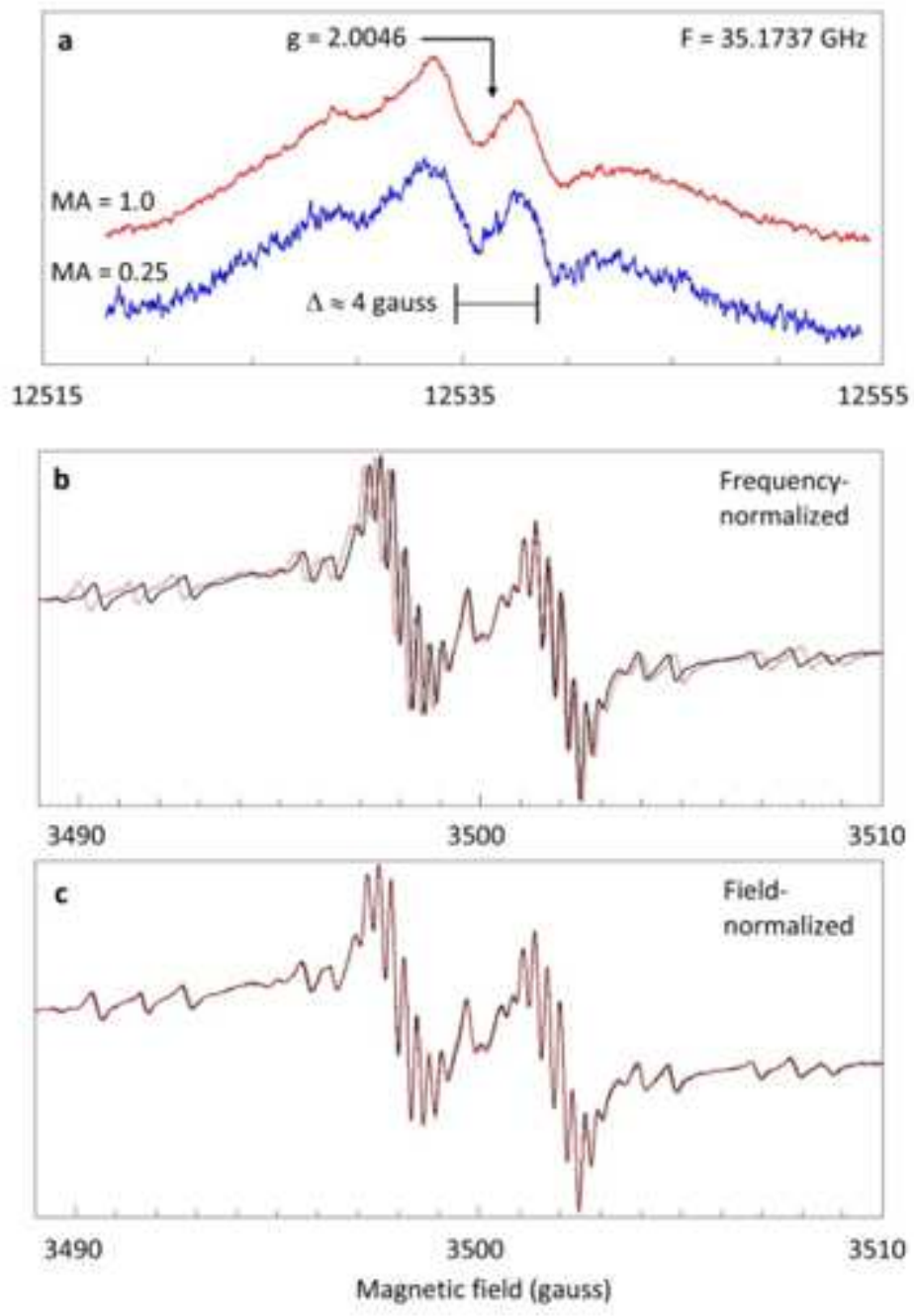

\section{Figure 3}

Multi-frequency experiments as checks on consistency of the EPR interpretation. Trace a: extensively averaged Q-band spectra taken at two different modulation amplitudes of 1 Gauss (red) or 250 mGauss (blue). No fine structure is resolved in addition to the two main lines consistent with an inhomogeneous line width linear in the microwave frequency. The central $g$ value and the splitting between the two lines in field units are identical to those observed in X-band. Trace b: extensively averaged intra X-band experiment at two frequencies, $9.4629 \mathrm{GHz}$ (red) and $9.8209 \mathrm{GHz}$ (black). Each field point of the red spectrum is frequency transformed to that of the black spectrum where the overlay shows that only the center of the two main lines is a real $g$ value. In trace $c$ the red spectrum is shifted in its entirety to a higher field for maximal overlap with the black spectrum. Here the overlay proves that there is only a 
single real $\mathrm{g}$ value and that all other features are constant in the field. See METHODS for experimental conditions.

\section{Supplementary Files}

This is a list of supplementary files associated with this preprint. Click to download.

- SupplementaryinformationHagenMills.docx 